\title{
Chandra Observations of NGC 4438: An Environmentally Damaged Galaxy in the Virgo Cluster
}

\author{
Marie E. Machacek, Christine Jones, and William R. Forman \\ Harvard-Smithsonian Center for Astrophysics, MS67, \\ 60 Garden Street, Cambridge, MA 02138 USA \\ mmachacek@cfa.harvard.edu, cjf@head-cfa.cfa.harvard.edu, \\ wrf@head-cfa.cfa.harvard.edu
}

\begin{abstract}
We present results from a $25 \mathrm{ksec}$ CHANDRA ACIS-S observation of galaxies NGC 4438 and NGC 4435 in the Virgo Cluster. X-ray emission in NGC 4438 is observed in $\mathrm{a} \sim 700 \mathrm{pc}$ nuclear region, $\mathrm{a} \sim 2.3 \mathrm{kpc}$ spherical bulge, and a network of filaments extending $4-10 \mathrm{kpc}$ to the $\mathrm{W}$ and $\mathrm{SW}$ of the galaxy. The X-ray emission in all three regions is highly correlated to similar features observed in $\mathrm{H} \alpha$. Spectra of the filaments and bulge are well represented by a $0.4 \mathrm{keV}$ MEKAL model with combined $0.3-2 \mathrm{keV}$ intrinsic luminosity $L_{\mathrm{X}}=1.24 \times 10^{40} \mathrm{erg} \mathrm{s}^{-1}$, electron densities $\sim 0.02-0.04 \mathrm{~cm}^{-3}$, cooling times of $400-700 \mathrm{Myr}$ and X-ray gas mass $\lesssim 3.7 \times 10^{8} M_{\odot}$. In the nuclear region of NGC $4438 \mathrm{X}$-ray emission is seen from the nucleus and from two outflow bubbles extending $360 \mathrm{pc}(730 \mathrm{pc})$ to the NW (SE) of the nucleus. The spectrum of the NW outflow bubble plus nucleus is well fit by an absorbed $\left(n_{H}=1.9_{-0.4}^{+1.0} \times 10^{21} \mathrm{~cm}^{-2}\right)$ $0.58_{-0.10}^{+0.04} \mathrm{keV}$ MEKAL plasma model plus a heavily absorbed $\left(n_{H}=2.9_{-2.0}^{+3.1} \times 10^{22} \mathrm{~cm}^{-2}\right)$ $\Gamma=2$, power law component. The electron density, cooling time, and X-ray gas mass in the NW outflow are $\sim 0.5 \mathrm{~cm}^{-3}, 30 \mathrm{Myr}$ and $3.5 \times 10^{6} M_{\odot}$. Weak X-ray emission is observed in the central region of NGC 4435 with the peak of the hard emission coincident with the galaxy's optical center; while the peak of the soft X-ray emission is displaced $416 \mathrm{pc}$ to the NE. The spectrum of NGC 4435 is well fit by a dominant, $\Gamma=1.7$, power law plus a weaker $\sim 0.2 \mathrm{keV}$ MEKAL component. We argue that the X-ray properties of gas outside the nuclear region in NGC 4438 and in NGC 4435 favor a high velocity, off-center collision between these galaxies $\sim 100 \mathrm{Myr}$ ago; while the nuclear X-ray emitting outflow gas in NGC 4438 has been heated only recently (within $\sim 1-2 \mathrm{Myr})$ by shocks $\left(v_{s} \sim 600 \mathrm{~km} \mathrm{~s}^{-1}\right)$ possibly powered by a central AGN.
\end{abstract}

Subject headings: galaxies: clusters: general — galaxies: individual (NGC 4438) intergalactic medium - X-rays: galaxies 


\section{Introduction}

It is well established that the stellar populations in galaxies in the cores of rich clusters have undergone substantial changes on relatively recent timescales $(z \lesssim 0.5)$. Butcher \& Oemler $(1978$, 1984) showed that clusters of galaxies at high redshift $(z>0.2)$ generally had a larger fraction of blue galaxies compared to that observed in nearby clusters. While morphologically these galaxies appeared to fall on the normal Hubble sequence, spectroscopically many of them were quite different from those observed in the present epoch. Dressler \& Gunn (1983) found that these blue galaxies belonged spectroscopically to three general classes: galaxies with ongoing star formation, galaxies containing active galactic nuclei (AGN) with characteristic broad and/or high-excitation line spectra, and E+A galaxies with strong Balmer absorption and little emission, characteristic of an early type galaxy in the post-starburst phase. Using the Hubble Space Telescope (HST), Dressler

et al. (1994) and Couch et al. (1998) found that most of the blue galaxies in distant clusters were of the first type, spirals or irregulars undergoing active star formation, and, as redshift decreased, these galaxies were found to increasingly avoid cluster cores. While the fate of these blue spiral and irregular galaxies is still debated, i.e. whether they simply fade from view or transform their morphological type, it is widely thought that their disappearance within rich cluster cores by the present epoch is driven by changes in their star formation rates and that the causes responsible for the observed evolution are more likely due to galaxy interactions with the cluster environment, predominantly within the dense cluster core, than to factors intrinsic to the galaxies themselves (e.g. Poggianti et al. 1999 and references therein).

A number of mechanisms for the interactions of galaxies within the rich cluster environment have been suggested. Morphological evidence from high resolution HST observations (Dressler et al. 1994; Couch et al. 1998) show that $20 \%$ of cluster galaxies, particularly those very blue galaxies undergoing or having just recently completed a starburst, show signatures of major mergers, i.e. interactions between galaxies of similar size, confirming the suggestion by Lavery \& Henry (1988) that in some cases mergers play an important role in triggering star formation and changes of morphological type. However, major mergers also are expected to completely disrupt the disk structure of the merger participants and yet disk structures persist, albeit many show evidence of structural distortion. For these galaxies some combination of minor mergers, galaxy-galaxy tidal interactions (Moore 1996), tidal interactions between the galaxy and the cluster potential (Byrd \& Valtonen 1990) and/or gas depletion mechanisms such as ram pressure stripping (Gunn \& Gott 1972; Bekki et al. 2002) and turbulent or viscous stripping (Nulsen 1982; Quilis et al. 2000) of the galaxy's interstellar medium (ISM) due to the motion of the galaxy through the hot, intracluster medium (ICM) are likely to be important. Furthermore, galaxies, in dynamically young clusters far from virialization, are more likely to show enhanced star formation or AGN activity than those in relaxed, virialized clusters (Couch et al. 1998; Ellingson et al. 2001; Miller 2003). This suggests that dynamical changes in the cluster potential or in the properties of the ICM due to subcluster mergers significantly affect the evolution of the galaxies moving through them. What is clear from the data is that no single mechanism is able to explain the observed transformation of galaxy colors 
and types in clusters. Rather, several processes may act in concert to deplete the gas content of galaxies, regulate their star formation, and drive their evolution.

Studies at a variety of wavelengths are essential in order to determine the relative contributions of the different physical processes acting on galaxies within clusters. Simulations show that different physical processes produce quite different density and temperature structures within the gas (Abadi et al. 1999; Quilis et al. 2000; Schulz \& Struck 2001; Vollmer et al. 2001). Thus high resolution $\mathrm{X}$-ray observations may help distinguish between competing scenarios. While it is not yet possible to measure detailed X-ray characteristics within blue galaxies in distant clusters, there are spiral and irregular galaxies in present-epoch clusters that appear to be analogs to the high redshift blue galaxies. In this paper we present high resolution X-ray results from a $25 \mathrm{ks}$ observation of one such galaxy, NGC 4438 in the Virgo Cluster, taken with the Chandra X-ray Observatory.

The Virgo Cluster is a particularly good laboratory in which to study the effects of cluster environment on galaxies. It is a fairly rich, nearby $(D=16 \mathrm{Mpc})$ cluster with a significant ICM and population of infalling spirals. It also is dynamically young, showing signs of imminent or ongoing merging between a small $\sim 1-3 \times 10^{13} M_{\odot}$ group associated with the elliptical galaxy M86 $\left(v=-244 \mathrm{~km} \mathrm{~s}^{-1}\right)$ falling from behind into the major $\left(\sim 2 \times 10^{14} M_{\odot}\right)$ cluster component associated with the dominant elliptical galaxy M87 $\left(v=1307 \mathrm{~km} \mathrm{~s}^{-1}\right)$ located at the cluster core (e.g. Schindler et al. 1999). NCG $4438\left(12^{h} 27^{m} 45.6^{s},+13^{\circ} 00^{\prime} 32^{\prime \prime}, \mathrm{J} 2000\right)$ is a large SA/0 peculiar spiral galaxy located in projection only $58^{\prime}(\sim 270 \mathrm{kpc})$ to the northwest of M87 in the direction of M86. Given its extremely low line of sight velocity $\left(v=71 \mathrm{~km} \mathrm{~s}^{-1}\right)$, NGC 4438 may well be associated with the M86 subcluster. The nearest galaxy (in projection) to NGC 4438 is NGC 4435, a compact SB galaxy with line of sight velocity $v=801 \mathrm{~km} \mathrm{~s}^{-1}$ located $4^{\prime} .3$ to the north and west.

NGC 4438 is the most environmentally "damaged" galaxy in the Virgo cluster. Its highly disturbed optical morphology shows stellar tidal debris displaced well out of the disk in the direction of NGC 4435. This general westward asymmetry is also found in all tracers of the interstellar gas. Chincarini \& de Souza (1985) observed a westward extension in $\mathrm{H} \alpha+[\mathrm{N} \mathrm{II}]$ emission. Higher resolution measurements (Keel \& Wehrle 1993, Kenney et al. 1995) found that this extension formed filamentary fingers of $\mathrm{H} \alpha+[\mathrm{N} \mathrm{II}]$ emission extending westward from the disk. Molecular gas identified by $\mathrm{CO}$ emission was found displaced $68^{\prime \prime}$ to the west (Combes et al. 1988), coincident with a bright region of $\mathrm{H} \alpha$ emission, as well as in the galactic center (Combes et al. 1988; Kenney et al. 1995). X-ray observations using the Einstein satellite (Kotanyi et al. 1983; Fabbiano et al. 1992) showed both compact emission in the center of the galaxy and extended emission to the west, as do $1.4 \mathrm{GHz}$ radio continuum maps by Hummel et al. (1983). What little cold HI gas that is detected lies in two major clumps west of the galactic nucleus, one $70^{\prime \prime}$ to the west (Cayatte et al. 1990) between two $\mathrm{H} \alpha$ filaments in a region of low X-ray surface brightness (Kenney et al. 1995), and the other about halfway between NGC 4438 and NGC 4435 (Hibbard \& van Gorkom 1990). Together these observations indicate highly disturbed interstellar gas characterized by a wide range of density and temperature. 
Several possible interaction scenarios have been proposed to explain the asymmetric, extended features seen in NGC 4438. One possibility is that the violent disruption of the gas could be caused by ICM-ISM interactions, such as ram pressure stripping, as NGC 4438 passes with high relative velocity $\left(\sim 1200 \mathrm{~km} \mathrm{~s}^{-1}\right)$ through the densest part of the ICM near the Virgo cluster core (Kotanyi et al. 1983; Chincarini \& de Souza 1985; Keel \& Wehrle 1993). Alternatively, Combes et al. (1988) suggested that the pattern of stellar tidal debris was more consistent with a high velocity collision between NGC 4438 and NGC 4435; while the western, asymmetric gas features could have been caused by ISM-ISM interactions in such a collision (Kenney et al. 1995).

The nuclear region in NGC 4438 is equally intriguing. On the basis of low $\mathrm{H} \alpha /[\mathrm{NII}]$ ratios, indicating that photoionization by hot stars is not the dominant source of ionization, and the observation of a weak broad component in the $\mathrm{H} \alpha$ emission line profile, denoting the presence of high velocity gas (Ho et al. 1997b), NGC 4438 was classified as having a LINER1.9 or dwarf Seyfert nucleus. However, if photoionization by an AGN dominates, the X-ray emission should be strongly correlated with the $\mathrm{H} \alpha$ emission in both the hard and soft energy bands (Ward et al. 1988; Koratkar et al. 1995). Although the nuclear region is a strong source of soft, extended X-ray emission, as observed with Einstein ( Kotanyi et al. 1983), ASCA (Terashima et al. 2000), and ROSAT HRI (Roberts \& Warwick 2000; Halderson et al. 2001), the ratio of the $2-10 \mathrm{keV}$ X-ray luminosity to the $\mathrm{H} \alpha$ luminosity was found to be anomalously low for its LINER classification (Terashima et al. 2000). Using both broadband and narrowband HST images of the central 18" of the galaxy, Kenney \& Yale (2002) found bipolar shells indicative of gas outflows straddling a compact $\left(1^{\prime \prime}\right)$ nuclear source. They observed that the low $\mathrm{H} \alpha /[\mathrm{NII}]$ ratios originated predominantly from the edges of the shells from shock heated gas produced by the outflows interacting with the surrounding ISM rather than from the nuclear source. A similarly complex morphology was found in 1.49 and $4.86 \mathrm{GHz}$ radio continuum maps (Hummel \& Saikia 1991) with elongated structures in general agreement with the optical shells and a weak radio source at the nucleus. The nature of the nuclear source that powers the observed gas outflows remained unclear. Some properties of the outflows were found consistent with those expected from a very compact nuclear starburst, while others were better characterized as originating from a true AGN.

In this paper we reexamine these issues using Chandra observations of NGC 4438 and NGC 4435. We study the interaction of NGC 4438 with its environment through X-ray measurements of the properties of the disturbed, extended gas. We also probe with high spatial resolution the $\mathrm{X}$-ray properties of the galactic center to clarify the nature of the nuclear source. This paper is organized in the following way. In Section 2 we detail the observations and the data reduction and processing procedures. In Section 3 we discuss our main results. First we exploit the spatial resolution of Chandra to compare the X-ray to optical morphology of the field containing both NGC 4438 and its companion galaxy NGC 4435 as a whole. Then in Section 3.1 we compare the X-ray properties of gas in the extended filamentary features westward of NGC 4438 to those of gas in the bulge. We also compare our X-ray results to other wavelength bands. We show that the $\mathrm{X}$-ray emission in the extended features, as well as the bulge, is well characterized by a $0.4 \mathrm{keV}$ 
plasma model. The extended X-ray features found asymmetrically out of the plane of the disk to the west and southwest are highly correlated with the $\mathrm{H} \alpha+[\mathrm{N} \mathrm{II}]$ emission filaments, supporting the ISM-ISM interaction hypothesis of Kenney et al. (1995) for their origin. In Section 3.2 we study the nuclear emission region consisting of two outflow shells and a compact nuclear source. We show that X-ray emission from the gas in the outflow is consistent with shock heating from a relatively recent outburst from the nucleus and that the data favor a highly obscured AGN over a compact starburst for the nuclear source. In Section 3.3 we investigate the X-ray emission from NGC 4438's companion galaxy NGC 4435. We find evidence in NGC 4435 for a power law nuclear source as well as a weak, cool extended thermal component. We briefly discuss the point source population of the field in Section 3.4 and summarize our results in Section 4. Unless stated otherwise, all fluxes are observed fluxes, while all luminosities are intrinsic, i.e. have been corrected for absorption. All errors are $90 \%$ confidence limits and coordinates are J2000. Taking the distance to NGC 4438 as that of the Virgo Cluster core (16.1 Mpc, Kelson et al. 2000), 1" corresponds to a distance scale of $77 \mathrm{pc}$.

\section{Observations and Analysis}

Our data consist of a $25 \mathrm{ksec}$ exposure of NGC 4438 taken by the Chandra X-ray Observatory on 29 January 2002. The data were obtained using the Advanced CCD Imaging Spectrometer array (ACIS, Garmire et al. 1992; Bautz et al. 1998) operating in very faint mode to improve background rejection. The focal plane temperature of the instrument was $-120^{\circ} \mathrm{C}$ throughout the observation. Our analysis of NGC 4438 is based mainly on data near the aim point from the back-illuminated CCD S3 chip, although the imaging and spectral analysis of nearby NGC 4435 utilized data from the S2 chip as well. Each CCD chip is a $1024 \times 1024$ pixel array where each pixel subtends $0^{\prime \prime} .492 \times 0^{\prime \prime} .492$ on the sky.

The data were analyzed using the standard X-ray processing packages, CIAO 2.3, FTOOLS and XSPEC 10.0. Cosmic ray contamination was minimized by filtering out events with bad grades $(1,5$, and 7$)$ and those tagged as bad by the VFAINT data acquisition mode due to significant flux in border pixels of the $5 \times 5$ event islands. The use of VFAINT mode improves background rejection by as much as a factor of 3 in the back illuminated $\mathrm{S} 3$ chip for the soft X-ray energies $(\sim 0.3 \mathrm{keV})$ important to this observation. Bad pixels and columns also were removed in the standard manner. The light curve was checked for flares, i.e. periods of anomalously high background, and none were found. Since the spectrum of NGC 4438 is known to be soft, special care was taken to model detector response at low energies. In addition to the standard modeling of instrument response functions for spectral analysis, we included corrections for the declining efficiency of the ACIS detector, significant for energies below $1 \mathrm{keV}$, due to the buildup of contaminants on the optical filter and explicitly checked our spectral fits for sensitivity to this correction.

Blank sky background sets appropriate for the date of observation were used in the imaging analyses (see http://cxc.harvard.edu/ciao/threads/acisbackground/). We applied the same clean- 
ing algorithms to the data that were applied to the background sets. This resulted in the additional removal of short intervals at the beginning and end of the observation, reducing the useful exposure time to $24,891 \mathrm{~s}$. Identical spatial and energy filters were applied to source and background data throughout, so that the background normalization is set by the ratio of exposure times. We checked this normalization by comparing count rates in the high energy (9-14 keV) band, where particle background is expected to dominate, to that predicted by the normalized blank field background sets and found that they differ by $\lesssim 6 \%$. We adopt this difference as our relative uncertainty in the background level for this method.

Blank sky background subtraction is, in most cases, the preferred background subtraction technique for ACIS-S observations of extended sources since it models correctly the variation of the background across the S3 chip. In the spectral analysis of the extended emission from NGC 4438, however, it is important to account for the additional background due to the presence of the hot Virgo intracluster gas. We separated the cluster ICM component from the non-nuclear extended galactic ISM emission in two ways. First (Method B) we used blank sky background subtraction and modeled the intracluster gas explicitly in our spectral fits by including a fixed MEKAL thermal plasma model component appropriate for cluster gas at the location of NGC 4438 , i.e $58^{\prime}$ (272 kpc) from M87. We used our current data from a 95" circular region centered at $\left(12^{h} 27^{m} 35.4^{s},+12^{\circ} 57^{\prime} 11^{\prime \prime}\right)$ on chip S3 away from the NGC 4438 emission and blank sky background subtraction to fix the cluster parameters. We found the cluster data well represented by a MEKAL plasma model with $k T=2.1 \pm 0.3 \mathrm{keV}\left(\chi^{2} /\right.$ dof $\left.=80.6 / 77\right)$ for fixed abundance $0.3 Z_{\odot}$ (Ohashi et al. 1998) and Galactic absorption $\left(2.64 \times 10^{20} \mathrm{~cm}^{-2}\right)$. The normalization of the cluster component in the spectral analyses of extended emission from NGC 4438 was fixed by the ratio of the areas of the galaxy to cluster extraction regions. We also used our model of the cluster gas to determine the cluster electron density in the vicinity of NGC 4438. Assuming a spherical $\beta$ model for the gas density distribution of the Virgo cluster with $\beta=0.47$ and core radius $2^{\prime} .7$, we found an electron density for the hot ICM local to NGC 4438 of $\sim 5.7 \times 10^{-4} \mathrm{~cm}^{-3}$. These Virgo cluster model parameters and the inferred cluster electron density are in general agreement with previous measurements (Ohashi et al. 1998, Schindler et al. 1999). Second (Method L) we took the same region used to fit the cluster gas as a local background region to directly subtract the background plus cluster emission. We examined blank sky background sets to verify that variations in the background observed between the source region and local background region were small, $\lesssim 3 \%$ for the $0.3-2 \mathrm{keV}$ energy range used in these spectral fits. We used the local background region to subtract both the standard background and Virgo cluster contribution and performed spectral fits to the outer extended emission from NGC 4438.

For the nuclear emission region, additional backgrounds consist of both the Virgo cluster gas and the outer extended emission from NGC 4438 itself. For this smaller extraction region, background variation across the S3 chip is not a concern. We used a local background annulus of $7^{\prime \prime} .4$ inner and $20^{\prime \prime} .7$ outer radii centered on the $5^{\prime \prime}$ nuclear region to separate the nuclear emission from the cluster and outer galactic components. 


\section{Results}

In Figure 1 we compare a Palomar DSS optical ( $\lambda 6450)$ image of a $6^{\prime} .6 \times 9^{\prime} .4$ region containing NGC 4438 and NGC 4435 (right panel) to the $0.3-2 \mathrm{keV}$ Chandra X-ray image (left panel) of the same region matched in WCS coordinates. The Chandra data have been smoothed with a $\sigma=2^{\prime \prime}$
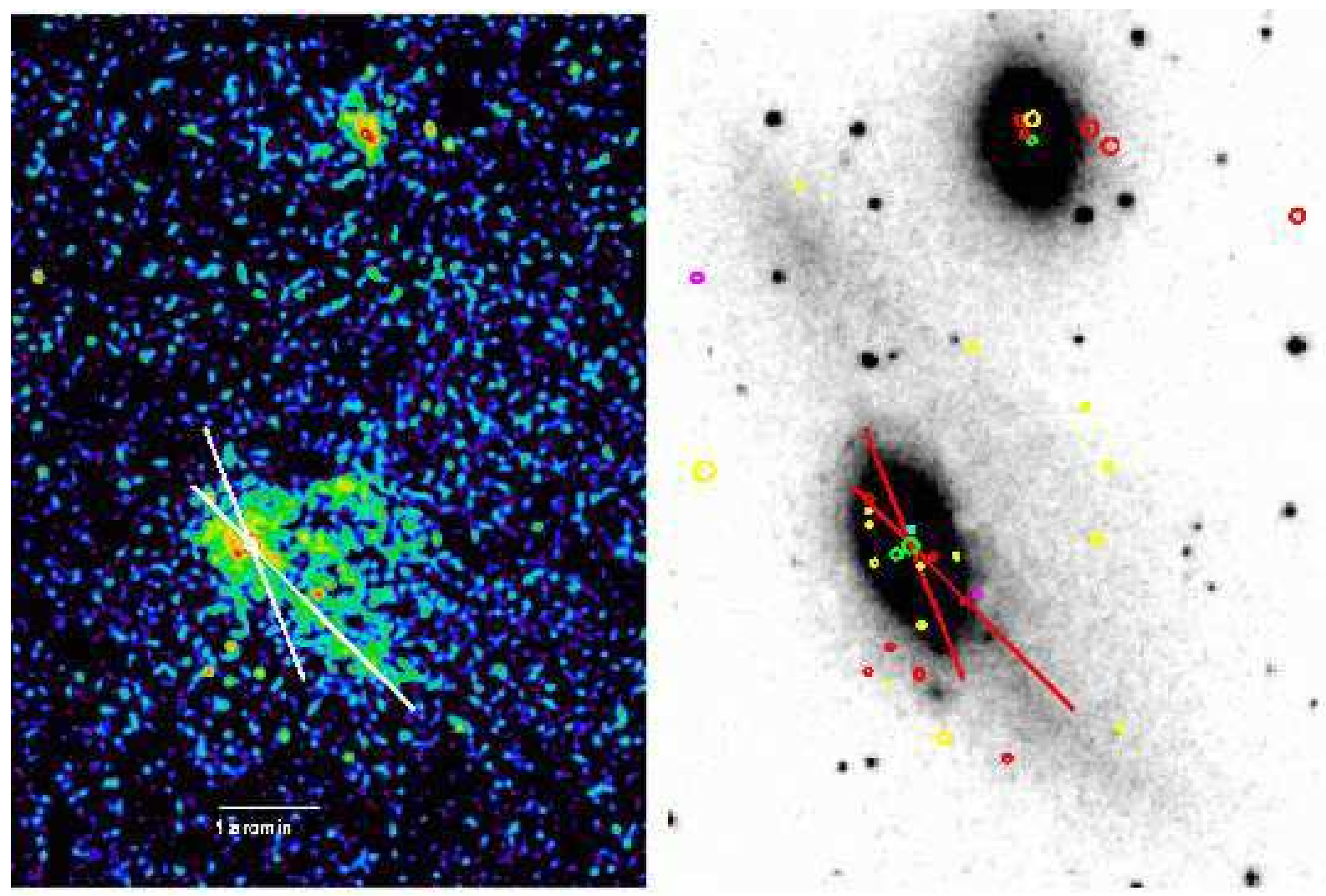

Fig. 1.- (left) Chandra $0.3-2 \mathrm{keV}$ band image of the $6^{\prime} .6 \times 9^{\prime} .4$ field containing galaxies NGC 4438 and NGC 4435. North is up and East is to the left. The X-ray image has been Gaussian smoothed $\left(\sigma=2^{\prime \prime}\right)$, background subtracted and exposure corrected. The image stretch is logarithmic from $4 \times 10^{-9}$ to $4 \times 10^{-6}$ photons $\mathrm{s}^{-1} \mathrm{~cm}^{-2} \operatorname{arcsec}^{-2}$. (right) DSS optical ( $\left.\lambda 6450\right)$ image of the same field taken with the Palomar 48 inch Schmidt telescope and matched in WCS coordinates with the Chandra image. The image stretch is linear from $1000-5000$ counts $\operatorname{arcsec}^{-2}$ and pixel size is $1^{\prime \prime} .7$. Broadband $(0.3-10 \mathrm{keV})$ X-ray point sources are superposed as circles on the optical image with colors denoting source strength in the hard $(2.0-10 \mathrm{kev})$ and soft $(0.3-2 \mathrm{keV})$ energy bands as follows: magenta denotes $\geq 10$ net counts in both the hard and soft bands; red (cyan) denotes $\geq 10$ net counts in the soft (hard) band but not both; yellow denotes $<10$ counts in either of the narrower bands. Green circles denote nuclear outflow bubbles in NGC 4438 and the hard nucleus in NGC 4435. Crossed lines show the directions of the major axis of NGC 4438 and the leading $\mathrm{X}$-ray filament. The horizonal angular scale bar represents $1^{\prime}$. 
Gaussian and background subtracted. Telescope vignetting and spatial efficiency variations have been corrected by means of an exposure map generated using standard CIAO tools assuming a fixed energy of $0.85 \mathrm{keV}$ corresponding approximately to the peak of the X-ray spectrum.

The highly disturbed morphology of NGC 4438 is apparent in both the optical and X-ray images in Figure 1. The optical image shows tidally disrupted stellar features extending $\sim 4^{\prime} .4$ to the northeast and $\sim 3^{\prime} .6$ to the southwest of the galactic center. An additional tidally distorted stellar fold makes an angle of $\sim 27^{\circ}$ with respect to the northeastern feature in the direction of NGC 4435; while the southwestern structure forms a nearly linear stellar ridge. The X-ray emitting gas is equally disturbed. The X-ray emission can be separated into three major components: a strongly emitting nuclear region, a roughly spherical (radius $\sim 2.3 \mathrm{kpc}$ ) bulge emission region surrounding the nucleus, and a network of filamentary features $\sim 1 \mathrm{kpc}$ thick extending $\sim 4-10 \mathrm{kpc}$ to the west, well out of the galactic disk. The leading (most southern) X-ray filament lies $\lesssim 8^{\prime \prime} .7$ west of the southwestern optical stellar tidal ridge and is oriented $\sim 23^{\circ}$ counterclockwise from the major axis of the galaxy as shown by crossed lines in both panels of Figure 1. We discuss the detailed properties of these diffuse filamentary structures in Section 3.1. The positions of two extended regions of X-ray emission located near the galactic center are superposed as green circles on the optical image. We will show in Section 3.2 that these regions correspond to the nuclear outflow regions observed by Kenney \& Yale (2002) in $\mathrm{H} \alpha+[\mathrm{N} \mathrm{II}]$ emission. NGC 4435 also shows asymmetric emission about its center that will be discussed further in Section 3.3. The locations of broadband $(0.3-10 \mathrm{keV})$ point sources identified by a multiscale wavelet decomposition algorithm are shown as circles in Figure 1, color coded to denote source strength. These will be discussed in Section 3.4.

\subsection{NGC 4438: The X-ray Filaments}

Diffuse X-ray emission from NGC 4438, as with other ISM tracers, is displaced to the west of the galaxy out of the plane of the disk. In Figure 2 we compare the X-ray emission from NGC 4438 to the $0^{\prime \prime} .77$ resolution $\mathrm{H} \alpha+[\mathrm{N} \mathrm{II}]$ map of Kenney et al. (1995) and find a striking correspondence between the X-ray and $\mathrm{H} \alpha+[\mathrm{N} \mathrm{II}]$ features. The $\mathrm{H} \alpha$ filaments 1, 2 and 3 (labeled clockwise in the left panel of Figure 2 from the northern-most filament as in Kenney et al. 1995), or equivalently the "bubble" (composed of filaments 1 and 2) and linear filament (filament 3) from Keel \& Wehrle (1993), coincide with similarly shaped X-ray features. Although we detect faint X-ray emission from a region coincident with the faint $\mathrm{H} \alpha$ filament 4 identified by Kenney et al. (1995), it appears patchy rather than forming a linear structure as seen in $\mathrm{H} \alpha$. We do see faint X-ray emission in the form of a short, linear extension (labeled filament $3 a$ ) similar to a weak $\mathrm{H} \alpha$ feature described by Kenney et al. (1995) roughly parallel to filament 3 beginning $\sim 13^{\prime \prime}$ to the northwest of that filament near a bright X-ray point source. We find, in agreement with previous Einstein HRI observations (Kotanyi et al. 1983), that the brightest region of X-ray emission in filament 1 , located $\sim 70^{\prime \prime}$ to the northwest of the nucleus, overlaps both the region of weak extended $\mathrm{CO}$ emission observed by Combes et al. (1988) and the dominant $\mathrm{H} \alpha+[\mathrm{N} \mathrm{II}]$ emission in this filament (Kenney et al. 1995). 

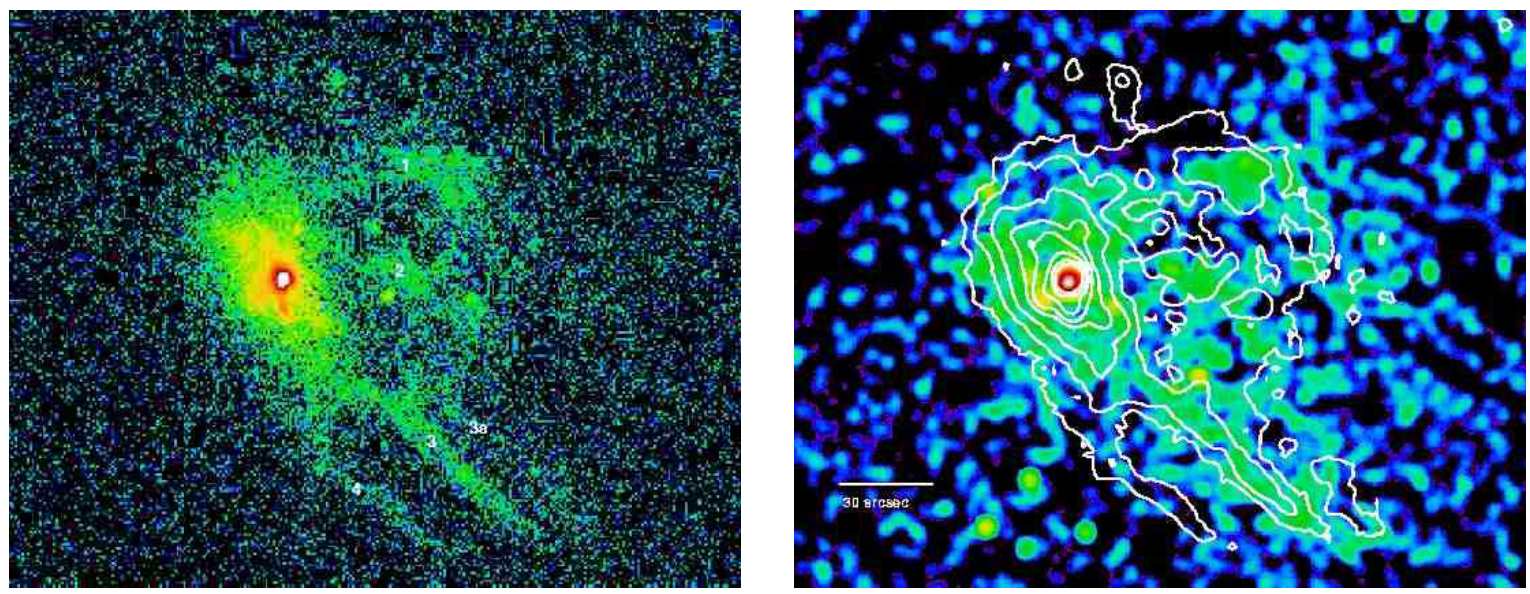

Fig. 2.- (left) KPNO H $\alpha+[\mathrm{N} \mathrm{II}]$ image of NGC 4438 (Kenney et al. 1995). The image stretch is logarithmic from $0.05 \%-100 \%$ of the peak value. Contours superposed on the Chandra image to the right are $0.0096 \%, 0.24 \%, 0.48 \%, 1.4 \%, 2.4 \%, 4.8 \%$ of the peak value. (right) Chandra image of NGC 4438 in the $0.3-2 \mathrm{keV}$ energy band with $\mathrm{H} \alpha$ contours superposed. The Chandra image has been Gaussian smoothed $\left(\sigma=2^{\prime \prime}\right)$, background subtracted and exposure corrected. The image stretch is logarithmic from $4 \times 10^{-9}$ to $4 \times 10^{-6}$ photons $\mathrm{s}^{-1} \mathrm{~cm}^{-2} \operatorname{arcsec}^{-2}$. The horizontal angular scale bar is $30^{\prime \prime}$ and in both images North is up and East is to the left.

\subsubsection{Mean Spectral Properties}

In order to obtain an average spectrum for the diffuse gas, we first fit the extended emission in the mean by choosing a circular extraction region $95^{\prime \prime}$ in radius centered at $\left(12^{h} 27^{m} 42.3^{s},+13^{\circ} 00^{\prime} 13^{\prime \prime}\right)$ containing the bulge and filamentary features, but with point sources and two circular regions of radius $4^{\prime \prime} .4$ and $3^{\prime \prime}$ containing the northwestern and southeastern nuclear outflow regions removed. Both blank sky and local background subtraction methods were used to check that our results for the emission from NGC 4438 were not dependent on our treatment of the Virgo cluster emission. Spectra were analyzed using XSPEC 10.0 with the data grouped to require a minimum of 20 counts per spectral bin. The results of these fits are summarized in Table 1.

When deriving the gas temperature and abundance for the diffuse emission using blank sky background data (Method B), we modeled the contribution of the hot Virgo cluster gas explicitly by including a MEKAL plasma emission model component with parameters fixed by our fit to the Virgo cluster gas at the radial distance of NGC 4438. A second MEKAL component was used to characterize the galactic emission and a common hydrogen absorbing column was assumed for both components. The absorbing column was initially fixed at the Galactic value $\left(2.64 \times 10^{20} \mathrm{~cm}^{-2}\right)$. The temperature, metallicity, and normalization of the second MEKAL component were fit (Model MEKAL). We restricted the energy range to $0.3-3 \mathrm{keV}$ where there were significant source counts compared to the background. A temperature $k T=0.37_{-0.02}^{+0.03} \mathrm{keV}$ and abundance $A=0.14_{-0.03}^{+0.05} Z_{\odot}$ (90\% CL) gave an acceptable fit to the mean spectrum of the diffuse X-ray emitting gas. We 
checked the stability of this fit by allowing the absorbing column to vary and show the results in Table 1 column 3. The temperature of the gas remains well determined $\left(k T=0.38_{-0.02}^{+0.03} \mathrm{keV}\right)$, although the absorbing column and abundances are less well constrained $\left(\mathrm{N}_{\mathrm{H}} \leq 3.6 \times 10^{20} \mathrm{~cm}^{-2}\right.$, $\left.A=0.18_{-0.07}^{+0.13} Z_{\odot}\right)$. The fits agree within their $90 \%$ confidence limits.

The total $0.3-2 \mathrm{keV}$ flux for this region is $4.93 \times 10^{-13} \mathrm{erg} \mathrm{s}^{-1} \mathrm{~cm}^{-2}$ with NGC 4438 contributing $68 \%\left(3.35 \times 10^{-13} \mathrm{erg} \mathrm{s}^{-1} \mathrm{~cm}^{-2}\right)$ and the cluster accounting for the remaining $32 \%(1.58 \times$ $10^{-13} \mathrm{erg} \mathrm{s}^{-1} \mathrm{~cm}^{-2}$ ) in this energy band. After correcting for Galactic absorption, the $0.3-2 \mathrm{keV}$ intrinsic luminosity of the diffuse emission at a distance of $16.1 \mathrm{Mpc}$ is $\mathrm{L}_{\mathrm{X}}^{\text {total }}=1.81 \times 10^{40} \mathrm{erg} \mathrm{s}^{-1}$ with $1.25 \times 10^{40} \mathrm{erg} \mathrm{s}^{-1}$ from NGC 4438 and $5.6 \times 10^{39} \mathrm{erg} \mathrm{s}^{-1}$ from the ICM component. Since the diffuse emission from NGC 4438 is soft, with only $\sim 1 \%$ of its flux above $2 \mathrm{keV}$, the $2-10 \mathrm{keV}$ luminosity is dominated by the hot cluster component. The diffuse gas in NGC 4438 contributes only $\sim 2 \%$ $\left(7.1 \times 10^{37} \mathrm{erg} \mathrm{s}^{-1}\right)$ of the total $2.9 \times 10^{39} \mathrm{erg} \mathrm{s}^{-1}$ hard band emission.

When emission from the Virgo cluster ICM is subtracted by selecting a local background region (Method L), the number of net "source" counts is reduced from 3390 to 2238 due to the elimination of the Virgo cluster emission. Since the diffuse regions of NGC 4438 contribute little to the X-ray emission above $2 \mathrm{keV}$, we restrict the energy band for these spectral fits to $0.3-2 \mathrm{keV}$ where the galaxy count rate is significantly above background. Holding the absorbing column fixed at the Galactic value, we find the spectrum for the mean outer diffuse gas from NGC 4438 is well fit by a single temperature MEKAL model with gas temperature $k T=0.42_{-0.04}^{+0.07} \mathrm{keV}$ and abundance $A=0.11_{-0.02}^{+0.05} Z_{\odot}$. This gives an X-ray flux in the $0.3-2 \mathrm{keV}$ energy band for the diffuse gas from

Table 1. Mean spectral fits to the diffuse gas in NGC 4438

\begin{tabular}{cccc}
\hline \hline Method/Model & B/MEKAL & B/MEKAL & L/MEKAL \\
\hline source counts & 3390 & 3390 & 2238 \\
energy (keV) & $0.3-3$ & $0.3-3$ & $0.3-2$ \\
$k T(\mathrm{kev})$ & $0.37_{-0.02}^{+0.03}$ & $0.38_{-0.02}^{+0.03}$ & $0.42_{-0.06}^{+0.07}$ \\
$A\left(Z_{\odot}\right)$ & $0.14_{-0.03}^{+0.05}$ & $0.18_{-0.07}^{+0.13}$ & $0.11_{-0.02}^{+0.05}$ \\
$\mathrm{~N}_{\mathrm{H}}\left(10^{20} \mathrm{~cm}^{-2}\right)$ & $2.64^{f}$ & $\leq 3.6$ & $2.64^{f}$ \\
$\chi^{2} /$ dof & $140 / 94$ & $139 / 93$ & $103 / 82$ \\
\hline
\end{tabular}

Note. - Method B (blank sky background subtraction) fits both cluster and galaxy components. Method L (local background subtraction) fits the galaxy component alone. Superscript $f$ denotes a fixed parameter. 
NGC 4438 of $3.35 \times 10^{-13} \mathrm{erg} \mathrm{s}^{-1} \mathrm{~cm}^{-2}$ corresponding to an intrinsic X-ray luminosity in this band of $1.24 \times 10^{40} \mathrm{erg} \mathrm{s}^{-1}$, in good agreement with the results of Method B.

We also tried fitting the data, using both Method B and Method L, with a variable MEKAL model where we divided the elements into two groups, the alpha elements $(\mathrm{O}, \mathrm{Mg}, \mathrm{Si}, \mathrm{Ca})$ and all others, and tied the element abundances in each group together in the fit. We then fit the temperature and each group of abundances, while keeping the absorbing column fixed at the Galactic value. Within their 90\% confidence limits, there was no significant difference in the abundance for alpha and non-alpha elements. Similarly the temperature and abundances agreed within the $90 \%$ confidence limits with those of the corresponding simple MEKAL model. We thus do not include them in Table 1.

\subsubsection{Individual Features}

To gain insight into the physical mechanisms responsible for the distorted morphology of NGC 4438, we compare the X-ray properties of the filaments to X-ray emitting gas in the bulge. We used a circular region of radius $30^{\prime \prime}(2.3 \mathrm{kpc})$ about the nucleus, excluding point sources and the nuclear outflow regions, to extract the bulge emission and found $566 \pm 26$ source counts in the $0.3-2 \mathrm{keV}$ energy band (Method L), sufficient for an independent spectral fit. Using a single temperature MEKAL model with fixed Galactic absorption, we found a temperature $k T=0.42_{-0.05}^{+0.09} \mathrm{keV}$ and abundance $A=0.12_{-0.04}^{+0.10} Z_{\odot}$, in complete agreement with the mean fits given in Table 1 . In Figure 3 we show spectra and the model fits for these two regions. Thus we found no significant difference in the temperature and abundances for gas in the bulge compared to that in the mean ( and thus the filamentary extended features), even though the bulge gas contributes only $\sim 25 \%$ of the total extended diffuse emission. Thirty-four percent of the total extended emission comes from the filaments/bubble, while the remaining $41 \%$ is from diffuse gas not associated with any feature.

In Table 2 we characterize the extended X-ray features by simple geometries, e.g a sphere for the bulge, cylinders (boxes in projection) for the filaments and a spherical shell for the "bubble". The extent of each feature is represented by the length scales for the assumed geometry, i.e. the radius of a sphere, (radius $r$, length $l$ ) for cylinders, and (inner, outer) radii for the spherical shell. The cylinders are assumed to be figures of rotation about their long axes, such that the line of sight extent of a filament is its diameter $2 r$. Except for the bulge, these individual features are too weak to independently constrain all the X-ray spectral parameters. Instead we fixed the temperature and abundance at the best fit values given in Table 1 for the mean and allowed the normalizations to vary to determine the X-ray flux and luminosity of each feature. The spectral normalizations were then used to determine the emission measure for each region and, assuming gas uniformly fills the assumed geometry, to infer an rms electron density $<n_{e}^{2}>^{1 / 2}$ and mass of X-ray emitting gas. The reader should note that if the gas does not uniformly fill the volume, the electron density is increased by a factor $\eta^{-1 / 2}$ and the mass is decreased by a factor $\eta^{1 / 2}$ where $\eta \leq 1$ is the filling factor for the region. In Table 2 we list the fluxes, luminosities, average electron densities and X-ray 

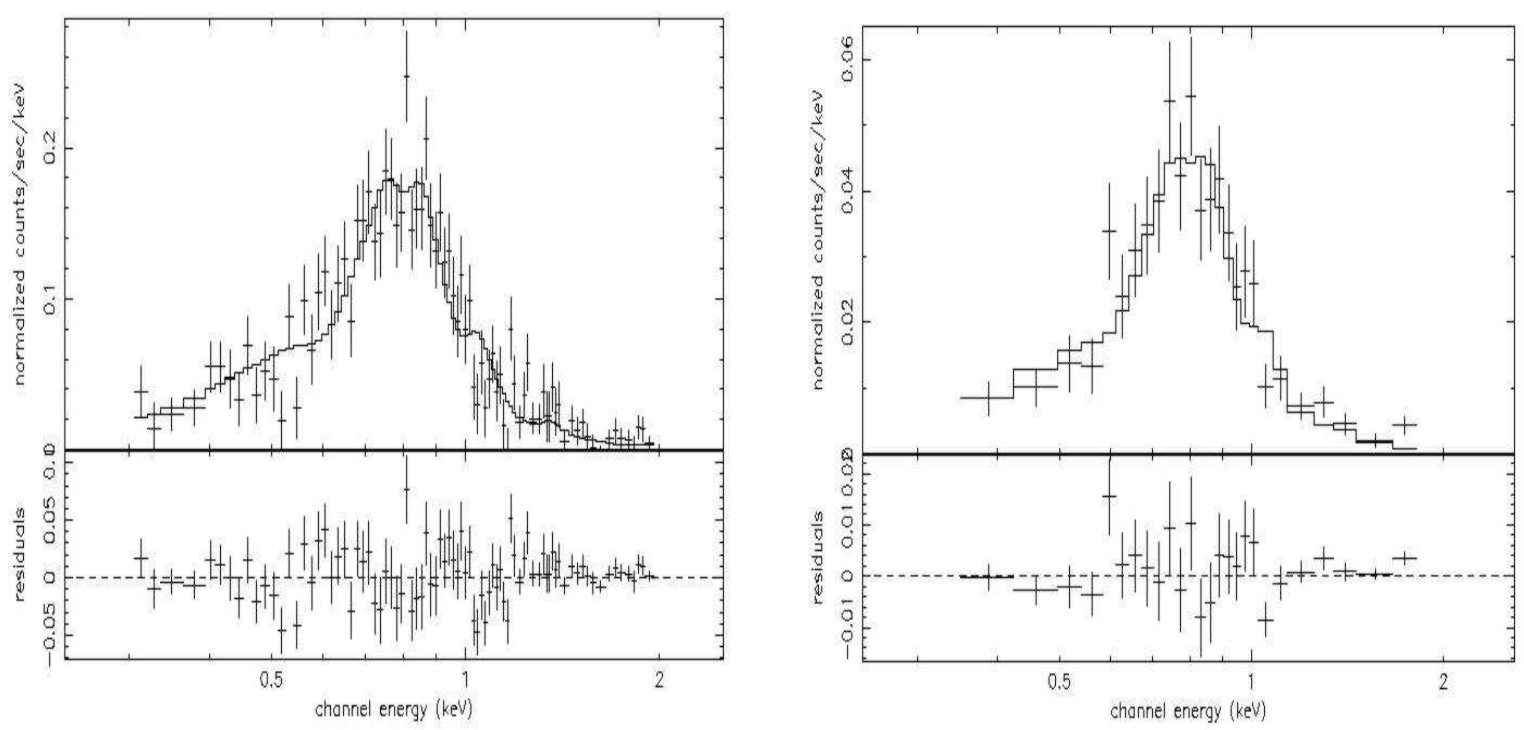

Fig. 3.- Spectra and best fit MEKAL models, using local background subtraction, for the extended diffuse gas in NGC 4438 in the mean (left) and in the bulge (right).

emitting gas mass for the mean emission, bulge emission, and emission from each filament/bubble. Since the spectral fits in Table 1 all agree within their $90 \%$ confidence limits, we list in Table 2 only the results for the fixed MEKAL model with local background subtraction. Errors on the inferred electron densities and hot gas masses are dominated by uncertainties in the assumed geometries for each feature, rather than by uncertainties in the spectral fits.

The rms electron densities for the X-ray emitting gas in the bulge and the filaments are found to be $\sim 0.02-0.04 \mathrm{~cm}^{-3}$, in agreement with estimates by Kotanyi et al. (1983). The lower electron density $\left(0.008 \mathrm{~cm}^{-3}\right)$ inferred from the diffuse emission as a whole (see the top row of Table 2) is a reflection of the fact that the X-ray emitting gas does not uniformly fill the volume, but much of it is distributed in the observed filamentary or bubble-like features. If we assume a filling factor of 1 for the $95^{\prime \prime}$ spherical volume as a whole, we find a conservative upper limit to the total extended X-ray emitting gas mass of $\lesssim 3.7 \times 10^{8} M_{\odot}$, a factor of 2.4 lower than the estimate by Kenney et al. (1995) using Einstein data and an approximate X-ray flux-to-mass conversion factor (Canizares et al. 1987). On the other hand, if we assume that gas only resides in the bulge and filamentary features, we derive a lower bound on the total hot gas mass in NGC 4438 of $\gtrsim 6-7 \times 10^{7} M_{\odot}$ (assuming uniform filling and the electron density derived for each feature) with $3.2 \times 10^{7} M_{\odot}$ still bound to the bulge and, depending on the chosen geometry, $3-4 \times 10^{7} M_{\odot}$ contained in the extended features. Optical emission line spectra (Kenney et al. 1995; Chemin et al. 2003) show that the velocities of the cooler, optically emitting gas in the extended filaments are within $200 \mathrm{~km} \mathrm{~s}^{-1}$ of the central velocity of NGC 4438. Assuming that the X-ray gas in each corresponding feature shares a common flow velocity with that of the cooler gas, the gas in these 
extended filaments may remain gravitationally bound to the galaxy.

Given the measured temperature $(\sim 0.4 \mathrm{keV})$, abundance $\left(A \sim 0.1-0.2 Z_{\odot}\right)$ and inferred electron density $\left(0.02-0.04 \mathrm{~cm}^{-3}\right)$ for both X-ray emitting gas in the extended filamentary features and in the bulge, we can determine its characteristic cooling time. The cooling function for this temperature and abundance is $\Lambda \sim 5 \times 10^{-24} \mathrm{erg} \mathrm{cm}^{3} \mathrm{~s}^{-1}$. Thus, the cooling time for gas at these densities,

$$
t_{\text {cool }}=\frac{3 n k T}{2 n_{e} n_{H} \Lambda(T)}
$$

where $n, n_{e}$, and $n_{H}$ are the gas, electron, and hydrogen number densities, $k$ is Boltzmann's constant and $T$ is the temperature, is $t_{\text {cool }} \sim 4-7 \times 10^{8} \mathrm{yr}$.

It is also interesting to note that, using our derived values for the electron densities in the cluster and the bulge, the thermal pressure of the X-ray emitting gas in the bulge is only a factor $\sim 1.5$ larger than the ram pressure on NGC 4438 due to its motion through the Virgo ICM. The contribution of the thermal pressure of the ICM is small compared to the contribution of the ram pressure at these velocities. Given the large uncertainties in the electron densities, this is consistent with local pressure equilibrium between the leading hot bulge gas and the ICM wind. We can also compare the thermal pressure between the cooler gas in the optical filaments and X-ray gas. Kenney et al. (1995) and Keel \& Wehrle (1993) infer electron densities in the optical filaments of $\sim 500 \mathrm{~cm}^{-3}$ and $<100 \mathrm{~cm}^{-3}$, respectively, using measurements of $[\mathrm{S} \mathrm{II}] \lambda \lambda 6716 / 6731$ doublet line ratios and an assumed temperature of $10^{4} \mathrm{~K}$ for the cool gas. Using these values and uniform filling factors, the thermal pressures of the $4.6 \times 10^{6} \mathrm{~K} \mathrm{X}$-ray gas and the $\sim 10^{4} \mathrm{~K}$ optically

Table 2. X-ray properties of extended emission in NGC 4438

\begin{tabular}{|c|c|c|c|c|c|c|c|}
\hline Region & Geometry & $\begin{array}{c}\text { Size } \\
(\operatorname{arcsec})\end{array}$ & $\begin{array}{c}\text { NGC } 4438 \\
\text { counts }\end{array}$ & $\begin{array}{c}\text { Flux } \\
\left(10^{-13} \mathrm{cgs}\right)\end{array}$ & $\begin{array}{c}\mathrm{L}_{\mathrm{X}}^{I S M} \\
\left(10^{40} \mathrm{erg} \mathrm{s}^{-1}\right)\end{array}$ & $\begin{array}{c}<n_{e}^{2}(\text { gal })>^{1 / 2} \\
\left(10^{-2} \mathrm{~cm}^{-3}\right)\end{array}$ & $\begin{array}{c}\mathrm{M}_{\text {gas }} \\
\left(10^{7} M_{\odot}\right)\end{array}$ \\
\hline mean & sphere & 95 & $2238 \pm 75$ & 3.35 & 1.24 & 0.8 & 37 \\
\hline bulge & sphere & 30 & $566 \pm 26$ & 0.83 & 0.31 & 2.3 & 3.23 \\
\hline filament 1 & cylinder & $(12,70)$ & $232 \pm 17$ & 0.37 & 0.14 & 2.8 & 1.15 \\
\hline filament 2 & cylinder & $(7.6,54)$ & $141 \pm 13$ & 0.22 & 0.08 & 3.9 & 0.50 \\
\hline bubble & shell & $(14,30)$ & $375 \pm 23$ & 0.57 & 0.21 & 1.9 & 2.58 \\
\hline filament 3 & cylinder & $(7.4,120)$ & $269 \pm 19$ & 0.40 & 0.15 & 3.6 & 0.98 \\
\hline filament 3a & cylinder & $(4.6,77)$ & $55 \pm 9$ & 0.08 & 0.03 & 3.4 & 0.23 \\
\hline filament 4 & cylinder & $(6,61)$ & $25 \pm 6$ & 0.05 & 0.02 & 2.3 & 0.22 \\
\hline
\end{tabular}

Note. - Size is characterized by radius for spheres, (inner, outer) radii for the shell, and (radius, length) for cylinders. The energy bandpass for flux and luminosity is $0.3-2 \mathrm{keV}$, except for filament $3 \mathrm{a}(0.36-2 \mathrm{keV})$ and filament $4(0.43-2 \mathrm{keV})$ due to XSPEC matrix bound limitations. Fluxes are observed. Luminosities are corrected for Galactic absorption. "cgs" denotes the flux unit $\mathrm{erg} \mathrm{s}^{-1} \mathrm{~cm}^{-2}$. 
emitting gas appear to differ. Equality of the thermal pressures given these assumptions would imply an electron density $\sim 20 \mathrm{~cm}^{-3}$ for the cool component. However, the uncertainties are large. In addition to uncertainties in the emission volume and filling factors for both components as well as the temperature of the cool gas, the dependence of the [S II] doublet line ratios on the electron density is weak in this low density regime such that it is not a sensitive discriminator between electron densities of a few 10's to a few $100 \mathrm{~cm}^{-3}$ (Osterbrock 1989). Thus it is plausible that local thermal pressure equilbrium is satisfied. Better optical as well as X-ray measurements would be needed to determine whether the thermal pressures are in fact discrepant, signaling either expansion of the optical filaments or the presence of non thermal pressure components.

Three striking results emerge from our analysis. First, we see the same extreme asymmetry in the X-ray gas as seen in other ISM tracers. X-ray emitting gas outside the bulge is concentrated to the south and west of the galaxy, while little emission is seen to the north. Second, the X-ray features extending out of the plane of the disk are in close correspondence to the $\mathrm{H} \alpha+[\mathrm{N}$ II] features observed by Keel \& Wehrle (1993) and Kenney et al. (1995), sharing the same angular positions and filamentary structure. This suggests that the filamentary features consist of a heterogeneous mixture of gas in two or more phases, i.e. $\mathrm{H} \alpha$ emitting gas at temperatures $\sim 10^{4} \mathrm{~K}$ and X-ray gas at temperatures $\sim 4.6 \times 10^{6} \mathrm{~K}$, as well as molecular gas in filament 1 (Combes et al. 1988). Finally, the properties of X-ray emitting gas in the filaments, i.e. temperature, metallicity, and density, are remarkably similar to those of X-ray gas in the bulge.

Two competing scenarios, both involving a combination of tidal and gas interaction processes, have been proposed to explain the complex morphology seen in NGC 4438. One scenario uses tidal interactions due to a close flyby of the galaxy with the Virgo cluster center to produce the observed stellar tidal tails (Miller 1986); while ram pressure and turbulent stripping of the ISM due to the motion of the galaxy through the intracluster medium are invoked to explain the trailing distribution of hot gas (Kotanyi et al. 1983). Simulations by Miller (1986) showed that tidal forces from the close approach of a disk galaxy to the cluster core would produce two long, oppositely directed stellar tails of various shapes that could include dense folds or hooks such as seen in Figure 1 in the northern stellar tidal feature in NGC 4438. Recent hydrodynamical simulations of ram pressure stripping in galaxies (Balsara et al. 1994; Schulz \& Struck 2001, Acreman et al. 2003) have shown that the gas dynamics of ICM-ISM stripping is complex. In particular, Schulz \& Struck (2001) showed in 3-D simulations of ICM-ISM interactions in disk galaxies that the stripped gas, while displaced from the disk, may be "hung up" downstream of the disk due to the increased gravitational influence of the dark matter halo on the displaced gas. They found that this might account for filamentary hydrogen gas structures with scales up to several galactic diameters. This is consistent with the fact that the longest filaments in NGC 4438 are roughly twice the diameter of the central gas bulge. Schulz \& Struck also found that the temperature of the gas in the wake depended on the initial density of the cloud. For moderate to high densities, cooling dominated heating by the ICM such that much of the gas cooled to temperatures $\sim 10^{4} \mathrm{~K}$. This could account for strong $\mathrm{H} \alpha$ emitting gas in the downstream features. Less dense clouds would be subject to 
heating by the ICM to temperatures of order that of the cluster gas and thus would be X-ray emitting. Using the criteria from Schulz \& Struck (2001) for NGC 4438 in Virgo, the critical electron density between these two regimes is $\sim 0.04 \mathrm{~cm}^{-3}$, consistent with the densities observed in the X-ray filaments. Tangled magnetic fields, either dragged out of the disk or produced by the dynamo effect due to turbulent motions induced by the stripping process, might inhibit thermal conduction between these clouds and delay their thermal mixing.

While qualitatively appealing, this scenario encounters several difficulties. First, as noted by previous authors (e.g Combes et al. 1988; Kenney et al. 1995), ram pressure stripping by the Virgo ICM is roughly two orders of magnitude too weak to displace the molecular gas observed in filament $168^{\prime \prime}$ from the inner disk. Second, recent simulations of ram pressure stripping (Abadi et al. 1999; Quilis et al. 2000; Schulz \& Struck 2001) all agree that the process is very prompt. Even in the filamentary structures "hung up" by the halo potential, Schulz \& Struck (2001) found that most of the gas would be stripped from the galaxy on a timescale of a few hundred million years. Thus stripping is expected to occur during the initial infall of the galaxy toward the cluster center and would most likely be complete before a close flyby of the cluster center could produce the observed tidal stellar tails. Furthermore, in the simulations, the temperature of gas shock heated by the ICM, with a relative velocity $\sim 1200 \mathrm{~km} \mathrm{~s}^{-1}$, tends to be much higher than the $0.4 \mathrm{keV}$ observed for diffuse gas in the extended filaments and the bulge of NGC 4438. Third, these same simulations showed that ram pressure also affects the structure of retained gas in the inner disk. In particular, this gas would suffer a bulk displacement from the dynamical center of the galaxy. Subsequent radial compression of the inner disk gas would result in the onset of gravitational instabilities and a ring of enhanced density and star formation on timescales of a few disk dynamical times, i.e. $\sim 1-3 \times 10^{8} \mathrm{yrs}$. These effects are not seen in the central region of NGC 4438. This suggests that the duration of gas-gas interactions in NGC 4438 was short, much less than the $\sim 10^{8}$ yr rotation period for gas in the bulge, so that gravitational instabilities would not have had time to develop and the effects of any disturbance of the retained gas in the central regions could have been erased. Finally while ram pressure caused by the motion of the galaxy through the ICM would produce characteristic asymmetries between the leading bow-shaped edge of the galaxy and the trailing gas wake, most simulations show symmetry of the wake about the central axis of the galaxy along its direction of motion. Thus it is more difficult to explain the paucity of stripped gas to the northwest, relative to that in the south. In addition, as noted by Kenney et al. (1995), if cluster tidal forces and ICM stripping are responsible for the observed morphology, it seems unlikely, given the number of other galaxies near the Virgo cluster core, that NGC 4438 alone should show such severe damage.

A more natural explanation for the observed features in NGC 4438 is that we are seeing the aftermath of a relatively high velocity, off-axis collision with nearby NGC 4435. Combes et al. (1988) were able to reproduce the observed stellar tidal tails and the northwest stellar fold in simulations of a retrograde collision $10^{8}$ years ago between NGC 4435 and NGC 4438 with relative velocity $\sim 900 \mathrm{~km} \mathrm{~s}^{-1}$ and point of closest approach $\sim 5 \mathrm{kpc}$ (about twice the radius of the X-ray emission region in the bulge) to the north of NGC 4438's optical center. Such a collision velocity 
is reasonable, given the observed $730 \mathrm{~km} \mathrm{~s}^{-1}$ line-of-sight relative velocity between the two galaxies and their close proximity in projection. The observed asymmetries in the tidal distortions are also easier to explain in the context of galaxy-galaxy collisions. The gravitational potential from the less massive NGC 4435 changes significantly over galaxy-sized scales, such that the tidal forces due to the collision would be significantly different on the near and far side of NGC 4438 from the collision point. This would produce strong distortions in the stellar distribution to the northeast and northwest closest to the collision path and a small angular displacement from the disk direction for the southwest stellar ridge (see Figure 1). In contrast, cluster induced tidal forces do not change significantly over galaxy scales and thus tend to produce more symmetrical tidal features (Byrd \& Valtonen 1990).

Similarly the asymmetric distribution of the extended X-ray filaments and their close correlation with the $\mathrm{H} \alpha+[\mathrm{N} \mathrm{II}]$ filaments argue for ISM-ISM interactions in an off-axis galaxy-galaxy collision to the north as their common origin (Kenney et al. 1995). In hydrodynamical simulations of ISM-ISM interactions in a single phase medium in off-axis galaxy collisions, Müller et al. (1989) found that the resulting properties of the displaced ISM strongly depended on the relative velocity between the two galaxies. They found that due to the lower kinetic energy in galaxy-galaxy collisions with relative velocities $v \lesssim 1000 \mathrm{~km} \mathrm{~s}^{-1}$, such as proposed here, cooling within the shock fronts could not be neglected. The maximum temperatures found for the disturbed gas in these simulations were only $\sim$ a few times $10^{6} \mathrm{~K}$, consistent with the low $\left(4.6 \times 10^{6} \mathrm{keV}\right)$ X-ray temperatures we observe in the bulge and extended filaments in NGC 4438. They also found, depending on the masses of the colliding partners and the impact parameter of the collision, that the displaced gas could remain bound to the galaxy. The low velocities observed for gas in the extended optical filaments in NGC 4438 (Kenney et al. 1995; Chemin et al. 2003) suggests that this may be the case, with gas in the filaments still bound to NGC 4438 and in the process of falling back onto the disk. The timescale over which ISM-ISM interactions are effective in this collision $\left(\lesssim 10^{7} \mathrm{yr}\right.$ depending on the scale heights of the colliding gas; Kenney \& Yale 1995) is small compared to the elapsed time since the collision. This explains the relatively undisturbed appearance of gas within the central bulge since, in contrast to the case of ICM-ISM interactions, it will have had a number of dynamical time periods since the gas interactions have ceased to recover. An off-axis collision of two galaxies naturally explains the observed north-south asymmetry seen in the gas. Without ISM-ISM interactions, gas should be tidally disrupted in roughly the same tidal streams as the stars. However, due to the stronger ISM-ISM interactions to the north near the point of closest approach between the galaxies' centers ( as well as the stronger tidal interactions as the centers pass each other), gas outside the bulge is efficiently swept from the region, explaining the lack of both $\mathrm{H} \alpha$ and X-ray emission there. ISM-ISM interactions are expected to be weaker to the south of the bulge, perhaps explaining the small $\left(\lesssim 8^{\prime \prime} .7\right)$ displacement of filament 3 from the southwestern tidal stellar ridge.

While suggestive, we do not expect the symmetric, single phase hydrodynamic simulations of Müller et al. (1989) to reproduce in detail the properties of a multi-phase ISM-ISM collision. 
Instead, we expect that (as in the ICM-ISM simulations of Schulz \& Stuck, 2001) the balance between heating and cooling in clouds of different densities would profoundly affect the properties of the gas we now observe. Clouds above the now higher $\left(\gtrsim 0.1 \mathrm{~cm}^{-3}\right)$ critical density cool efficiently to $10^{4} \mathrm{~K}$, accounting for the strong $\mathrm{H} \alpha$ emission in the filaments. This is consistent with the higher densities now observed in the $\mathrm{H} \alpha$ gas (Kenney et al. 1995). X-ray emissions come from gas streams of initially lower density that are heated by the ISM-ISM interaction, also consistent with the lower densities we measure for this component. Due to the low metallicity as well as low temperatures found for this gas in our spectral fit, the cooling time for this gas $\left(\sim 4-7 \times 10^{8}\right.$ yrs $)$ is much longer than the $10^{8}$ yrs since the collision. Thus we do not expect that much of this gas would have cooled.

ISM-ISM ram pressures in a collision between NGC 4438 and NGC 4435 are expected to be 1 to 3 orders of magnitude larger than the corresponding ISM-ICM ram pressures from the motion of NGC 4438 through the Virgo cluster gas making it easier to explain how ISM-ISM interactions in concert with tidal forces could displace dense molecular gas $68^{\prime \prime}$ out from the center of the disk in filament 1 (Combes et al. 1988). The close correspondence of the X-ray features seen in our high angular resolution Chandra images of NGC 4438 to those seen in $\mathrm{H} \alpha$ emission by Kenney et al. (1995) suggests that these two heterogeneous gas phases coexist in the same filamentary structures. Although not required, magnetic fields may also have been dragged out of the disk and tangled due to turbulence produced in the collsion. Such a network of magnetic fields would both link the different gas phases and delay their thermal mixing. In such a case the forces binding multiphase gas to the disk would depend upon an average surface gas density over some larger region, rather than the local surface gas density within a particular cloud. (Kenney et al. 1995). Since these average densities typically tend to be an order of magnitude lower than the local density found within dense molecular clouds, this would further enhance the ability of ISM-ISM interactions to drag dense, molecular gas out of the disk along with the less dense ISM material during the collision.

\subsection{NGC 4438: The Nuclear Emission Region}

In Figure 4 we focus on the structure of the inner nuclear region of NGC 4438. To obtain a sharper Chandra image of the innermost nuclear region, we reprocessed our data to remove the standard pixel randomization. In the left panel of Figure 4, we show the resulting soft band $(0.3-2 \mathrm{keV})$ image of the nuclear region smoothed with a $0^{\prime \prime} .5$ Gaussian. The X-ray emission can be separated into a dominant elongated northwest feature, containing the peak X-ray emission and the nucleus, and a weaker feature located $\sim 9^{\prime \prime}$ to the southeast. In the middle panel, we show the $0^{\prime \prime} .1$ resolution HST H $\alpha$ ( $\left.\lambda 6563\right)$ image taken by Kenney \& Yale (2002) matched to the X-ray image using WCS coordinates. Strong correlations exist between these nuclear X-ray features and the outflow bubbles observed in $\mathrm{H} \alpha+[\mathrm{N} \mathrm{II}]$ with HST by Kenney \& Yale (2002). The northwestern X-ray feature corresponds to the northwest outflow bubble seen in the middle panel of Figure 4 with the peak X-ray emission near the southeastern tip, $\lesssim 0^{\prime \prime} .7$ from the location of the compact 

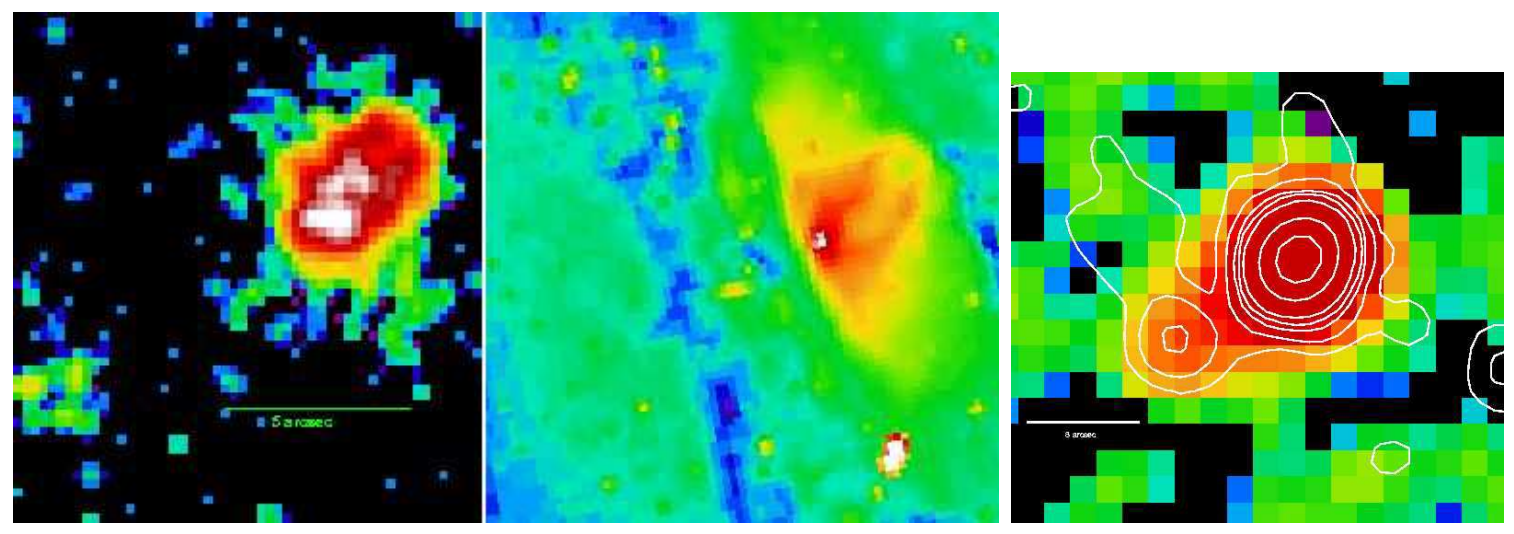

Fig. 4.- (left) $14^{\prime \prime} \times 14^{\prime \prime}$ nuclear region in the $0.3-2 \mathrm{keV}$ energy band with pixel randomization removed and smoothed with a $\sigma=0^{\prime \prime} .5$ Gaussian. The scale bar is $5^{\prime \prime}$. (middle) HST image ( $\lambda 6563$, Kenney \& Yale,2002) of the same region matched in WCS coordinates to the X-ray image on the left. (right) VLA First image of the $32^{\prime \prime} \times 32^{\prime \prime}$ field surrounding the nuclear region of NGC 4438, superposed with contours from a $\sigma=1^{\prime \prime}$ Gaussian smoothed, background subtracted, and exposure corrected Chandra X-ray image of the nuclear region. Contour levels are 0.4, 0.8, 1.6, 2.5, 4, 12, $25 \times 10^{-7}$ photons $\mathrm{s}^{-1} \mathrm{~cm}^{-2} \operatorname{arcsec}^{-2}$. The scale bar in the right panel is $8^{\prime \prime}$. In all three images North is up and East is to the left.

optical nucleus. While not readily visible in the $\mathrm{H} \alpha$ image alone, the $\mathrm{X}$-ray feature $\sim 9^{\prime \prime}$ to the southeast of the nucleus corresponds to the southeastern outflow shell identified by Kenney \& Yale (2002) in the continuum subtracted sum of $\mathrm{H} \alpha+[\mathrm{N} \mathrm{II}]$ HST images. This X-ray emission resolves into a ring-like pattern similar to the less organized, patchy emission observed in $\mathrm{H} \alpha+[\mathrm{N} \mathrm{II}]$. We, however, observe no X-ray emission $\left(\mathrm{L}_{\mathrm{X}} \lesssim 7.4 \times 10^{36} \mathrm{erg} \mathrm{s}^{-1}\right)$ from the location of the strong HII regions seen $8^{\prime \prime}$ south of the nucleus in the middle panel of Figure 4.

In the right panel of Figure 4 we superpose Chandra X-ray contours from a lower resolution 1" Gaussian smoothed, background subtracted, exposure corrected Chandra image of the nuclear emission region in the $0.3-2 \mathrm{keV}$ energy band on a $\sim 5^{\prime \prime}$ resolution $20 \mathrm{~cm}$ FIRST Survey map of the core region (see http://sundog.stsci.edu/). The X-ray emission is also strongly correlated to the radio continuum emission with the northwestern and southeastern nuclear X-ray peaks coinciding with radio continuum peaks A and B reported by Hummel \& Saikia (1991). These correlations between X-ray, $\mathrm{H} \alpha$ and radio features suggest a common origin for the emissions in shocks and postshock outflow gas, after the gas impacts the surrounding ISM.

We used a $5^{\prime \prime}$ circular region centered at $\left(12^{h} 27^{m} 45.6^{s},+13^{\circ} 00^{\prime} 32.8^{\prime \prime}\right)$ to study the spectral properties of the northwest outflow region, including the nuclear source. We chose a local background annulus with inner radius $7^{\prime \prime} .4$ and outer radius $20^{\prime \prime} .7$ centered on the source region to subtract the backgrounds due to the outer galactic diffuse emission and Virgo cluster gas. After background subtraction, the nuclear (northwest outflow) region had $1120 \pm 34$ net source counts in 
the $0.3-5 \mathrm{keV}$ energy band used in the spectral fits. We again fit spectra using XSPEC with a minimum of 20 counts per spectral bin. We used four different models each with a variable absorbing column, i.e. a single component power law, single component MEKAL, variable MEKAL, and power law plus MEKAL model, to probe the properties of the nuclear outflow gas and a possible nuclear source. These fits are summarized in Table 3.

A single, absorbed power law is unable to describe the spectrum, yielding an unphysical photon index $\Gamma=9.0$ and unacceptable $\chi^{2}$. A reasonable fit is obtained for a highly absorbed single temperature MEKAL model, with absorbing column $N_{H}=2.0_{-0.5}^{+0.4} \times 10^{21} \mathrm{~cm}^{-2}$, temperature $k T=$ $0.60 \pm 0.04 \mathrm{keV}$, and abundance $A=0.14_{-0.03}^{+0.06} Z_{\odot}$. The fit is not significantly improved by using a variable MEKAL model and allowing the alpha and non-alpha element abundances to vary independently. A good fit is obtained using two components, a highly absorbed power law to model the nucleus and an absorbed, single temperature MEKAL component to model emission from the outflow bubble. We fix the photon index at 2.0, consistent with an absorbed power law fit to the data above $2 \mathrm{keV}$. Such a steep spectral index $(\Gamma \sim 2)$ is expected in low luminosity advection dominated accretion flow (ADAF) systems (e.g. Narayan \& Yi 1995), where X-rays are produced by inverse Comption scattering of synchrotron photons off flow electrons, and has recently been used to model the nucleus in such systems as M87 (Di Matteo et al. 2003) and NGC 4594 (Pelligrini et al. 2003). The abundance of the outflow gas $\left(A=0.14 Z_{\odot}\right)$ is taken from the single temperature MEKAL model fit to the region and is similar to that found in the nuclear outflow gas in M51, a nearby Seyfert 2, spiral galaxy (Terashima \& Wilson 2001). We then allow the gas temperature

Table 3. Northwest Nuclear Outflow Region Spectra

\begin{tabular}{ccccc}
\hline \hline Model & PL & MEKAL & VMEKAL & PL + MEKAL \\
\hline $\mathrm{N}_{\mathrm{H}}^{a}\left(10^{22} \mathrm{~cm}^{-2}\right)$ & - & $0.20_{-0.05}^{+0.04}$ & $0.19_{-0.04}^{+0.05}$ & $0.19_{-0.04}^{+0.10}$ \\
$k T(\mathrm{kev})$ & - & $0.60_{-0.04}^{+0.04}$ & $0.61_{-0.05}^{+0.04}$ & $0.58_{-0.10}^{+0.04}$ \\
$A\left(Z_{\odot}\right)$ & - & $0.14_{-0.03}^{+0.06}$ & $0.11_{-0.04}^{+0.10}$ & $0.14^{f}$ \\
$A \alpha\left(Z_{\odot}\right)$ & - & - & $0.15_{-0.10}^{+0.21}$ & - \\
$\mathrm{N}_{\mathrm{H}}^{b}\left(10^{22} \mathrm{~cm}^{-2}\right)$ & 1.13 & - & - & $2.9_{-2.0}^{+3.1}$ \\
$\Gamma$ & 9.0 & - & - & $2.0^{f}$ \\
$\chi^{2} /$ dof & $182 / 40$ & $62 / 39$ & $61.5 / 38$ & $39.6 / 38$ \\
\hline
\end{tabular}

Note. - Model components are power law (PL), MEKAL, and variable MEKAL (VMEKAL). Fits include the nuclear source. $\mathrm{N}_{H}^{a}$ $\left(\mathrm{N}_{H}^{b}\right)$ are hydrogen column densities for the thermal (power law) components. Superscript $f$ specifies that the parameter is held fixed. 


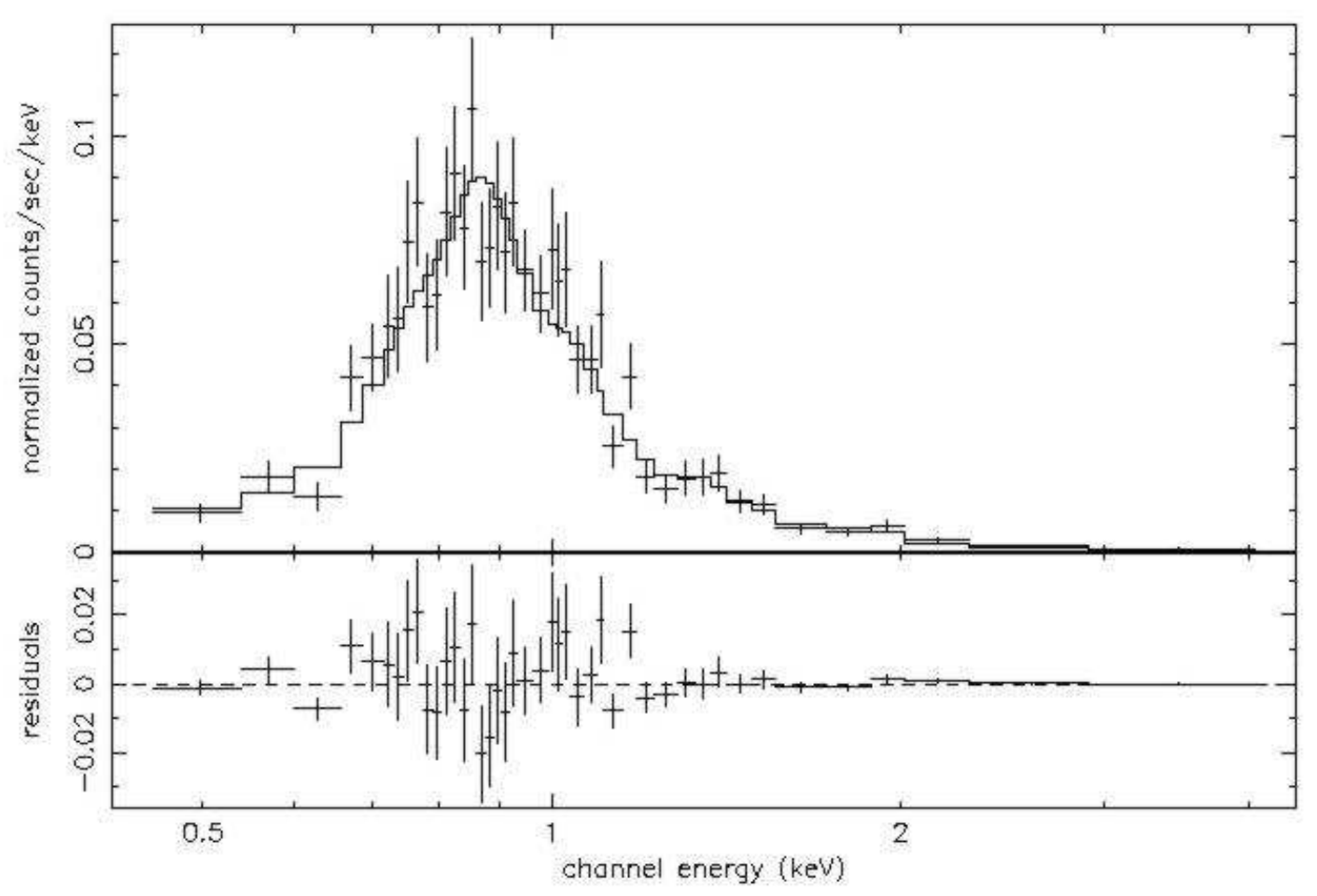

Fig. 5.- Spectrum and best fit absorbed powerlaw plus absorbed MEKAL model for the nucleus and northwest nuclear outflow region of NGC 4438.

and both absorbing columns to vary. We find the data are well fit with a gas temperature and absorbing column for the outflow component of $k T=0.58_{-0.10}^{+0.04} \mathrm{keV}$ and $n_{H}=1.9_{-0.4}^{+1.0} \times 10^{21} \mathrm{~cm}^{-2}$, consistent at the $90 \%$ confidence level with the MEKAL and VMEKAL fits listed in Table 3, and a moderately obscured $\left(n_{H} \sim 2.9_{-2.0}^{+3.1} \times 10^{22} \mathrm{~cm}^{-2}\right)$ power law component for the nuclear source. We show the spectrum and this best fit absorbed power law plus absorbed MEKAL model in Figure 3.2 .

Although the steep spectral index and location of the nucleus at the center of the galaxy are suggestive of a true AGN, we do not expect to be able to detect the fluorescent Fe $\mathrm{K}$ line in our spectrum due to our limited exposure. However, the Fe $\mathrm{K}$ line is also not seen in the analysis of ASCA data for NGC 4438 by Terashima et al. (2002). While the detection of the Fe K alpha line would clearly point to an AGN as the nuclear source, its absence, if verified by deeper observations, might, for example, merely denote the absence of the standard thin accretion disk, not the nuclear black hole. This may be common for low-luminosity AGN systems (for example, see NGC 4594, Pellegrini et al. 2003).

The fitted column densities for the nucleus and outflow gas are consistent with optical ex- 
tinction measurements of the region. Ho et al. (1997a) used $\mathrm{H} \alpha / \mathrm{H} \beta$ line ratios in a $2^{\prime \prime} \times 4^{\prime \prime}$ aperture about the nucleus (thus including contributions from the northwest outflow shell) to infer a weighted, mean intrinsic color excess $E(B-V)$ for this region of 0.61 . Assuming a normal dust to gas ratio, such that

$$
\frac{n_{\mathrm{H}}}{E(B-V)}=5.28 \times 10^{21} \mathrm{~cm}^{-2}
$$

(Binney \& Merrifield 1998, eq. 3.65), this color excess implies a mean intrinsic hydrogen column $n_{\mathrm{H}} \sim 3.2 \times 10^{21} \mathrm{~cm}^{-2}$ (total hydrogen column $n_{\mathrm{H}} \sim 3.5 \times 10^{21} \mathrm{~cm}^{-2}$ ). This column is consistent with, although marginally higher than, our fitted value for absorption of the outflow (thermal) component and a factor $3-17$ (given our large uncertainties) less than our fit to the absorption of the nuclear (power law) component. This is to be expected since, as pointed out by Kenney \& Yale (2002), the observed $\mathrm{H} \alpha+[\mathrm{N} \mathrm{II}]$ flux from the northwest outflow shell is 7 times that of the nucleus and the determination of the extinction corrections by Ho et al. (1997a) included a significant contribution from outflow gas as well as the nucleus. Thus our measured values are reasonable. Similarly the temperature we find for the outflow gas $\left(0.58_{-0.10}^{+0.04} \mathrm{keV}\right)$ is similar to temperatures measured for gas surrounding nuclei in other active galaxies such as M51 (0.5 keV,Terashima \& Wilson 2001) and NGC 4594 (0.6 keV, Pellegrini et al. 2003).

Fluxes and luminosities for the northwest outflow region as a whole and for the thermal (outflow gas) and power law (nuclear source) components separately are listed in Table 4 for the soft $(0.3-2 \mathrm{keV})$ and hard $(2-10 \mathrm{keV})$ energy bands. The soft band, where we have the most sensitivity, is dominated by thermal emission from the outflow gas, whose soft band observed flux and absorption-corrected luminosity are $1.4 \times 10^{-13} \mathrm{erg} \mathrm{s}^{-1} \mathrm{~cm}^{-2}$ and $1.0 \times 10^{40} \mathrm{erg} \mathrm{s}^{-1}$. If we assume that this gas uniformly fills a sphere of radius $5^{\prime \prime}$ (the size of the spectral extraction region), we infer a mean electron number density and mass of hot gas in the northwest outflow region of $<n_{e}^{2}>^{1 / 2} \sim 0.5 \mathrm{~cm}^{-3}$ and $3.5 \times 10^{6} M_{\odot}$. The thermal pressure of the $0.6 \mathrm{keV} \mathrm{X}$-ray gas at this density is comparable to that in the $10^{4} \mathrm{~K}$ gas $\left(n_{e} \sim 300-400 \mathrm{~cm}^{-3}\right.$, Kenney \& Yale 2002), consistent within the large uncertainties with local pressure equilibrium between these two components.

Table 4. Nuclear Region Observed Fluxes and Intrinsic Luminosities

\begin{tabular}{ccccc}
\hline \hline Component & $\begin{array}{c}\text { flux }(0.3-2.0 \mathrm{keV}) \\
\left(10^{-13} \mathrm{erg} \mathrm{s}^{-1} \mathrm{~cm}^{-2}\right)\end{array}$ & $\begin{array}{c}\mathrm{L}_{\mathrm{X}}(0.3-2.0 \mathrm{keV}) \\
\left(10^{39} \mathrm{erg} \mathrm{s}^{-1} \mathrm{~cm}^{-2}\right)\end{array}$ & $\begin{array}{c}\text { flux }(2.0-10.0 \mathrm{keV}) \\
\left(10^{-13} \mathrm{erg} \mathrm{s}^{-1} \mathrm{~cm}^{-2}\right)\end{array}$ & $\begin{array}{c}\mathrm{L}_{\mathrm{X}}(2.0-10.0 \mathrm{keV}) \\
\left(10^{39} \mathrm{erg} \mathrm{s}^{-1} \mathrm{~cm}^{-2}\right)\end{array}$ \\
\hline thermal & 1.41 & 10.2 & 0.08 & 0.26 \\
power law & 0.03 & 2.4 & 0.51 & 2.04 \\
total & 1.43 & 12.6 & 0.59 & 2.3 \\
\hline
\end{tabular}

Note. - Values above assume the absorbed power law plus MEKAL model given in Table 3. Fluxes are observed. Luminosities are corrected for absorption. 
Using equation 1 and a cooling function $\Lambda \sim 6.6 \times 10^{-24} \mathrm{erg} \mathrm{cm}^{3} \mathrm{~s}^{-1}$, the cooling time for the X-ray outflow gas is only $\sim 3 \times 10^{7} \mathrm{yr}$. Thus heating of the outflow gas must have occurred quite recently.

There are too few counts in the southeast outflow shell (35 \pm 6 in a circle of radius $\left.1^{\prime \prime} .7\right)$ to obtain a separate spectrum for that region. If we assume that the southeast outflow shell has the same temperature and absorbing column as the northwest outflow gas, we estimate its observed $0.3-2$ $\mathrm{keV}$ flux and intrinsic luminosity to be $\sim 4.8 \times 10^{-15} \mathrm{erg} \mathrm{s}^{-1} \mathrm{~cm}^{-2}$ and $\sim 3.4 \times 10^{38} \mathrm{erg} \mathrm{s}^{-1}$. This also implies an average electron density and cooling time of $\left\langle n_{e}^{2}>^{1 / 2} \sim 0.5 \mathrm{~cm}^{-3}\right.$ and $t_{\text {cool }} \sim 3 \times 10^{7} \mathrm{yr}$. Because of the low luminosity of the southeast outflow region, it is more difficult to define the bubble geometry and infer the X-ray gas mass. We estimate the mass of hot gas in this structure to be at least $1.3 \times 10^{5} M_{\odot}$. However, it could possibly be as high as that found in the northwest outflow structure.

Kenney \& Yale (2002) found that the optical line emission responsible for the LINER classification of NGC 4438 originated from shocked gas at working surfaces, where outflow gas interacts with the surrounding interstellar medium. If shocks also are responsible for heating the X-ray gas, we can use the outflow gas temperature to determine the bulk outflow velocity. The expected post-shock temperature $T_{s}$ of a fully ionized gas with $10 \%$ helium to hydrogen in number density is (Hollenbach \& McKee 1979)

$$
T_{s}=1.4 \times 10^{5} V_{7}^{2}
$$

where $V_{7}$ is the shock velocity in units of $10^{7} \mathrm{~cm} \mathrm{~s}^{-1}$. Therefore the shock velocity needed to heat gas to $0.5-0.6 \mathrm{keV}$ in the nuclear region is $600-700 \mathrm{~km} \mathrm{~s}^{-1}$. Kenney \& Yale (2002) find evidence from $[\mathrm{N} \mathrm{II}]$ and $[\mathrm{S} \mathrm{II}]$ line widths for line of sight gas velocities $\sim 300 \mathrm{~km} \mathrm{~s}^{-1}$. They argue that the true outflow velocities could be much higher. Thus to shock heat the gas would require an outflow velocity only a factor of $\sim 2$ higher than that measured along the line of sight.

The sizes of the outflow regions and their cooling times are consistent with the outflow having traveled from the nucleus in the recent past. The northwest outflow bubble has a maximum extent of $\lesssim 4^{\prime \prime} .7$ (360 pc). Even assuming the conservative lower limit to the outflow velocity $\left(300 \mathrm{~km} \mathrm{~s}^{-1}\right)$ measured by Kenney \& Yale (2002), the outflow could have originated in the nucleus $1.2 \times 10^{6}$ years ago, much less than the calculated cooling time of $\sim 3 \times 10^{7} \mathrm{yr}$ for gas in this bubble. The southeastern outflow bubble is twice as far from the nucleus $\left(9^{\prime \prime} .5\right.$ or $\left.\sim 730 \mathrm{pc}\right)$ and much fainter, consistent with it being an older structure with the nuclear outburst occurring twice as long ago as that which formed the northwestern outflow. This is still much less than the cooling time $\sim 3 \times 10^{7} \mathrm{yr}$ for the gas. It is also of order the synchrotron lifetime for electrons in the corresponding radio feature found by Hummel \& Saikia (1991), thus confirming the need for high outflow velocities $\sim 700 \mathrm{~km} \mathrm{~s}^{-1}$ and explaining the weakness of the radio source.

An alternative explanation for the different extent and brightness of the two outflow bubbles could be that the outflows represent bubbles of the same age but in different stages of evolution. This might be the case for outflows propagating through ISM of different densities. The SE outflow structure propagating into less dense material would then be assumed to be in the adiabatic or free- 
expanding phase such that it would radiate only weakly; while the NW outflow shell would be in the superbubble stage of evolution and would radiate more efficiently due to its rapid deceleration by denser ISM material (Kenney \& Yale 2002).

In either case, the energetics of the outflow gas provides insight into the nature of the nuclear power source. The amount of hot, ionized gas in the northwest outflow region $\left(3.5 \times 10^{6} M_{\odot}\right)$ is more than a factor of 30 greater than the lower limit placed on the gas by the $\mathrm{H} \alpha$ measurements alone (Kenney \& Yale 2002). Assuming the measured lower limit on the outflow velocity of $300 \mathrm{~km} \mathrm{~s}^{-1}$, the kinetic energy contained in this gas is $\sim 3 \times 10^{54} \mathrm{ergs}$, close to the kinetic energy found in the ionized gas outflow of NGC $3079\left(2 \times 10^{54} \mathrm{ergs}\right.$, Veilleux et al. 1994; Cecil et al. 2002) and greater than the $\sim 10^{53}$ ergs lower limit estimated by Kenney \& Yale (2002) for NGC 4438. If we assume that the nuclear source is a compact starburst, this amount of kinetic energy would require the energy output of more than 3000 supernova from a region $\lesssim 77$ pc in radius and, following the arguments of Kenney \& Yale (2002), a star formation rate $\gtrsim 0.3 M_{\odot} \mathrm{yr}^{-1}$ within the inner $77 \mathrm{pc}$ of NGC 4438. This star formation rate is above the upper limits on star formation for a nuclear starburst in this galaxy, derived from measurements of the $\mathrm{H} \alpha$ emission $\left(0.05 M_{\odot} \mathrm{yr}^{-1}\right)$ and the radio continuum power $\left(0.1 M_{\odot} \mathrm{yr}^{-1}\right)$ (Kenney \& Yale 2002). If we use the more realistic outflow velocity $\gtrsim 600 \mathrm{~km} \mathrm{~s}^{-1}$ from the shock heating analysis, the kinetic energy in the outflow gas could be a factor of 4 higher requiring a star formation rate in excess of $1 M_{\odot} \mathrm{yr}^{-1}$. Such a large kinetic energy in the outflow gas coupled with the measured low values for the nuclear star formation rate favors a central AGN, either alone or in addition to nuclear star forming activity, as the driver of the outflows. It is perhaps interesting to note that the kinetic pressure from this outflow $\left(1-4 \times 10^{-9}\right.$ dyne $\left.\mathrm{cm}^{-2}\right)$ is comparable to the thermal pressure $\left(\sim 10^{-9}\right.$ dyne cm $\left.^{-2}\right)$ of the $0.6 \mathrm{keV}$ gas. An AGN interpretation for the nuclear source also could explain the high degree of collimation in the southeastern bubble, the enhanced radio brightness observed at the ends of the outflow shells, and the weak $2050 \mathrm{~km} \mathrm{~s}^{-1}$ broad-line component observed in the nuclear spectrum by Ho et al. (1997b), but may require the AGN jets to be subrelativistic and thus not easily observed (Kenney \& Yale 2002; Bicknell et al. 1998).

Our best fit spectral model for the northwest outflow region does include a weak, power law component that could be attributed to an AGN nuclear source. However, this power law component is heavily absorbed, contributing predominately to the high energy tail of the observed spectrum. The power law plus MEKAL spectral model shown in Table 3 predicts a $2.0-10 \mathrm{keV}$ intrinsic luminosity for the nuclear source of $2 \times 10^{39} \mathrm{erg} \mathrm{s}^{-1}$. For this spectral index, the bolometric luminosity is about a factor of 10 larger (see, e.g. Pellegrini et al. 2003), $L_{\mathrm{bol}} \sim 2 \times 10^{40} \mathrm{erg} \mathrm{s}^{-1}$. However, due to our limited statistics at high energies, the errors, particularly on the absorbing column, are quite large. This translates into $\sim 60 \%$ uncertainty in the intrinsic luminosity of the nuclear source. If we assume, as did Kenney \& Yale (2002), a black hole mass of $5 \times 10^{7} M_{\odot}$ suggested for NGC 4438 by the black hole mass - bulge luminosity relation (Ferrarese \& Merrit 2000; Gebhardt et al. 2000), the ratio of the bolometric luminosity to the Eddington luminosity for the black hole is extremely small, $L_{\mathrm{bol}} / L_{\mathrm{Edd}} \sim 3 \times 10^{-6}$. Such sub-Eddington accretion appears to be common 
in most nearby galaxies (Roberts \& Warwick 2000). This ratio is similar to that found in NGC 4594 where the weakness of the source is attributed to radiatively inefficient or unsteady accretion (Pellegrini et al. 2003). Unsteady accretion onto a black hole in NGC 4438 might both account for the nuclear outflows and reconcile the energy budget for the flows, since the nuclear AGN would be episodic and could have been more energetic in the recent past.

In the likelihood that the nuclear source is an AGN, then the $2-10 \mathrm{keV}$ X-ray and $\mathrm{H} \alpha$ luminosities should obey the $L_{\mathrm{X}}-L_{H \alpha}$ relation

$$
\log L_{\mathrm{X}}=(1.11 \pm 0.054) \log L_{H \alpha}-(3.50 \pm 2.27)
$$

(Feigelson \& Babu 1992; Ho et al. 2001; see also Terishima et al. 2000 for a slightly different fit). Previous measurements of the $L_{\mathrm{X}} / L_{H \alpha}$ ratio in NGC 4438 were anomalously low, in part due to the inability to separate the contribution of the nuclear source from that of the outflow regions. Using high resolution HST measurements to resolve the nucleus and extinction corrections derived from measurements of $\mathrm{H} \alpha / \mathrm{H} \beta$ line ratios by $\mathrm{Ho}$ et al. (1997), Kenney \& Yale (2002) found the unabsorbed $\mathrm{H} \alpha$ luminosity of the nuclear source was $L_{H \alpha} \sim 5.5 \times 10^{39} \mathrm{erg} \mathrm{s}^{-1}$. Using the $2-10 \mathrm{keV}$ unabsorbed luminosity for the power law component in our best spectral fit for the X-ray luminosity of the nuclear source, $L_{\mathrm{X}} \sim 2 \times 10^{39} \mathrm{erg} \mathrm{s}^{-1}$, gives $\log \left(L_{\mathrm{X}} / L_{H \alpha}^{1.11}\right)=-4.8$, consistent with Equation 4. However, given the large uncertainties in the X-ray luminosity for the power law component, due to our limited statistics, and also in the extinction corrections for the $\mathrm{H} \alpha$ emission, the $L_{\mathrm{X}} / L_{H \alpha}$ ratio could be much lower.

\subsection{NGC 4435}

Figure 6 shows the central X-ray emission from NGC 4435. The X-ray morphology of this galaxy is asymmetric and knot-like. The brightest region of X-ray emission occurs in an elliptical region with major and minor axes of $6^{\prime \prime}$ and $4^{\prime \prime}$, similar in extent to the $3^{\prime \prime} .6$ optical emission-line region reported by Kenney et al. (1995). Emission in the $2-6 \mathrm{keV}$ energy band is dominated by a point source, the nucleus, located at the optical center of NGC 4435 (Argyle \& Clements 1990). There is a linear extension of hard emission northeast of the nucleus. However, since NGC 4435 is located $3^{\prime} .9$ off-axis from the telescope aim point, this may be an artifact of the point spread function at such large off-axis angles. Specifically, for this observation, the point spread function drops to $10 \%$ of its peak value on a $2^{\prime \prime} .5 \times 1^{\prime \prime} .4$ elliptical contour about its center at the location of NGC 4435. The peak of the soft $0.3-2 \mathrm{keV}$ X-ray emission is displaced $5^{\prime \prime} .4$ to the northeast of the nucleus and weaker emission is observed extending outward, generally away from the direction of NGC 4438 , to $\sim 25^{\prime \prime}-30^{\prime \prime}$ from the central core.

We find $349 \pm 23$ source counts in the $0.3-6 \mathrm{keV}$ energy band in a circular region of radius $32^{\prime \prime}$ centered on the nucleus of the galaxy. Background is subtracted using a local annular region of inner radius $36^{\prime \prime}$ and outer radius $72^{\prime \prime}$, also centered on the nucleus. The spectrum, shown in Figure 7 , clearly shows a hard tail extending to energies $\gtrsim 5 \mathrm{keV}$. Although there are too few 


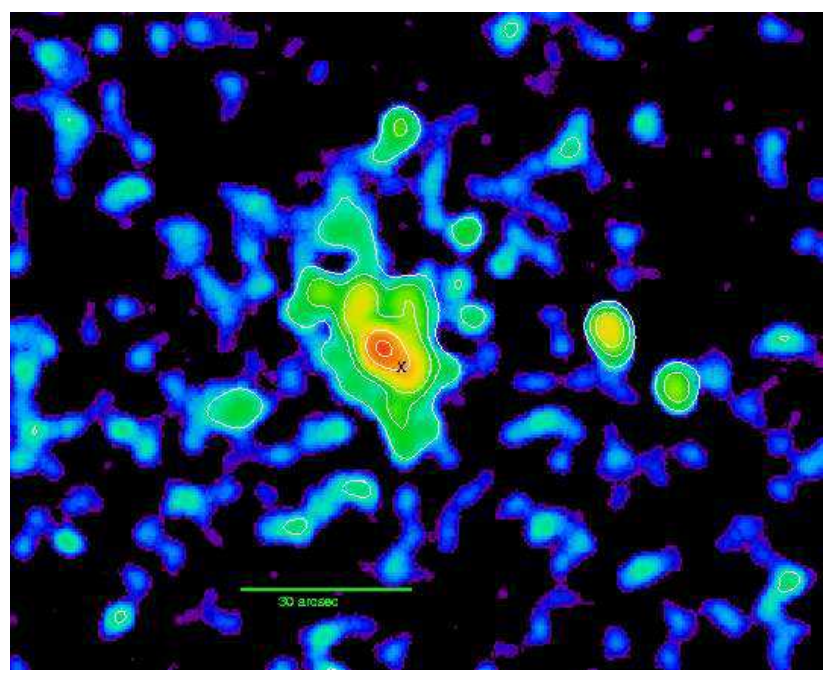

Fig. 6.- Chandra image of NGC 4435 in the $0.3-2 \mathrm{keV}$ energy band. The image has been smoothed with a $\sigma=2^{\prime \prime}$ Gaussian, background subtracted and exposure corrected. Contours are $1.24,2.48,4.13,12.4,20.7$ in units of $10^{-8}$ photons $^{-1} \mathrm{~cm}^{-2} \operatorname{arcsec}^{-2}$. X locates the peak of the $2-6 \mathrm{keV}$ hard band emission and the optical center of the galaxy. North is up and East is to the left. The horizontal scale bar is $30^{\prime \prime}(2.3 \mathrm{kpc})$.

counts for a detailed spectral fit, a simple power law spectral model with fixed Galactic absorption $\left(N_{H}=2.64 \times 10^{20} \mathrm{~cm}^{-2}\right)$ gives a photon index $\Gamma=1.9_{-0.3}^{+0.4}\left(\chi^{2} /\right.$ dof $\left.=37.9 / 21\right)$. Such a large photon index, typical for AGN, and the location of the hard emission at the galaxy's center are consistent with the identification of the nuclear source as a low luminosity AGN. This model gives a reasonable fit to the hard component above $1 \mathrm{keV}$, but underestimates the flux at low energies. The fit is improved by adding a thermal MEKAL model component, consistent with the presence of cool gas, in addition to the strong nuclear source. Assuming a fixed photon index $\Gamma=1.7$ (chosen to lie within the $90 \%$ confidence limits of the single power law fit), Galactic absorption, and abundance $A=0.1 Z_{\odot}$ similar to that found for NGC 4438 while allowing the temperature and both normalizations of the components to vary, we find a temperature for the cool gas of $k T=0.23_{-0.07}^{+0.09} \mathrm{keV}\left(\chi^{2} /\right.$ dof $\left.=26.3 / 20\right)$. The observed fluxes for the sum of both components in the soft $(0.4-2 \mathrm{keV})$ and hard $(2-10 \mathrm{keV})$ bands are $6.5 \times 10^{-14} \mathrm{erg} \mathrm{s}^{-1} \mathrm{~cm}^{-2}$ and $8.1 \times 10^{-14} \mathrm{erg} \mathrm{s}^{-1} \mathrm{~cm}^{-2}$ for this model, corresponding to intrinsic soft and hard X-ray luminosities of $2.3 \times 10^{39} \mathrm{erg} \mathrm{s}^{-1}$ and $2.5 \times 10^{39} \mathrm{erg} \mathrm{s}^{-1}$, respectively, at a distance of $16.1 \mathrm{Mpc}$. The power law (AGN) component dominates the emission with $0.4-2 \mathrm{keV}$ and $2-10 \mathrm{keV}$ fluxes of $4.4 \times$ $10^{-14} \mathrm{erg} \mathrm{s}^{-1} \mathrm{~cm}^{-2}$ and $8.1 \times 10^{-14} \mathrm{erg} \mathrm{s}^{-1} \mathrm{~cm}^{-2}$. The corresponding soft and hard band non-thermal intrinsic luminosities are $1.5 \times 10^{39} \mathrm{erg} \mathrm{s}^{-1}$ and $2.5 \times 10^{39} \mathrm{erg} \mathrm{s}^{-1}$. This corresponds to a bolometric luminosity of $\sim 2.5 \times 10^{40} \mathrm{erg} \mathrm{s}^{-1}$, comparable to the nonthermal nuclear source in NGC 4438 . The thermal component only has a significant contribution at low energies. Its $0.4-2 \mathrm{keV}$ observed flux and intrinsic luminosity are $2.1 \times 10^{-14} \mathrm{erg} \mathrm{s}^{-1} \mathrm{~cm}^{-2}$ and $7.6 \times 10^{38} \mathrm{erg} \mathrm{s}^{-1}$. Assuming that the 
gas uniformly fills the $32^{\prime \prime}$ spherical extraction volume, we use the normalization of the thermal component to find an electron density $<n_{e}^{2}>^{1 / 2}=0.015 \mathrm{~cm}^{-3}$, corresponding to a total hot gas mass of $2.8 \times 10^{7} M_{\odot}$.

On the basis of narrow optical emission line ratios, Ho et al. (1997a) classified NGC 4435 as a transition object, i.e. a galaxy with nuclear emission line properties intermediate between an AGN and an HII region. They proposed that the most natural explanation for these line ratios would be emission from a nuclear AGN, contaminated by emission from nearby HII regions. If this is the case and the nuclear region is also the site of recent star formation, the metallicity of the gas may be much higher. In order to determine how sensitive our results are to the assumed value for the abundance, we varied the abundance between $0.1 Z_{\odot}$ and $1.0 Z_{\odot}$. We found that the temperature and total soft band fluxes varied by $\lesssim 5 \%$, while the total hard band fluxes varied by $\lesssim 18 \%$. However, the normalization of the thermal component decreased with increasing abundance by a factor of $\sim 10$ between $A=0.1 Z_{\odot}$ and $1.0 Z_{\odot}$ which would reduce our estimates of the electron density and hot gas mass each by as much as a factor $\sim 3$. Thus $M \sim 3 \times 10^{7} M_{\odot}$ should be viewed as an upper limit on the X-ray emitting gas mass in NGC 4435. Combined with estimates of the atomic and molecular gas (each $\lesssim 4 \times 10^{7} M_{\odot}$, Kenney et al. 1995), this gives a total gas mass in NGC 4435 of $\lesssim 10^{8} M_{\odot}$.

We also checked our sensitivity to the chosen Galactic absorption. Ho et al. (1997a) measured an intrinsic color index $E(B-V)=0.68$ for the inner region of NGC 4435 using the $H \alpha / H \beta$ line ratios. Assuming a normal dust to gas ratio (Equation 2), this would imply a total (intrinsic plus Galactic) hydrogen absorption column $n_{\mathrm{H}}=3.85 \times 10^{21} \mathrm{~cm}^{-2}$. Using this optically motivated absorbing column in the above absorbed power law + MEKAL model spectral fits for NGC 4435, we find the temperature of the thermal component $\left(k T=0.17_{-0.04}^{+0.02}\right.$ for $A=0.1 Z_{\odot} ; k T=0.18 \pm 0.04$ for $A=1.0 Z_{\odot}$ ) agrees well with our previous fits, using only Galactic absorption, with no improvement in $\chi^{2}$. The total observed flux is also in good agreement. The soft $(0.4-2.0 \mathrm{keV})$ and hard $(2-10 \mathrm{keV})$ band intrinsic luminosities of the non-thermal component are each increased by $\sim 33 \%$. The most significant increase is, as expected, in the normalization and inferred absorption corrected luminosity of the thermal component. The soft band intrinsic luminosity increased by a factor $\sim 18$ and the electron density ( and thus upper limit on the gas mass) increased by a factor $\sim 5$. These measured values are still consistent with the gas mass and density estimates by Kenney et al. (1995).

In Figure 7 we show the spectrum and two extremes of these fits. In the left panel we show the best fit absorbed power law + MEKAL model with fixed $A=0.1 Z_{\odot}$ and Galactic absorption only; while in the right panel we show the best fit of the same model with fixed solar abundances and absorption $n_{\mathrm{H}}=3.85 \times 10^{21} \mathrm{~cm}^{-2}$ inferred from the measurements of optical extinction. The reader should note that the effects of increasing the abundance and also increasing the intrinsic absorption act oppositely on the electron density and hence the gas mass. For example, for the extreme case of solar abundances and high intrinsic absorption, the electron density $\left(n_{e} \sim 0.025 \mathrm{~cm}^{-3}\right)$ and total X-ray gas mass $\left(M \lesssim 4.7 \times 10^{7} M_{\odot}\right)$ are only a factor of 1.7 greater than that in the low abundance model assuming only Galactic absorption. 

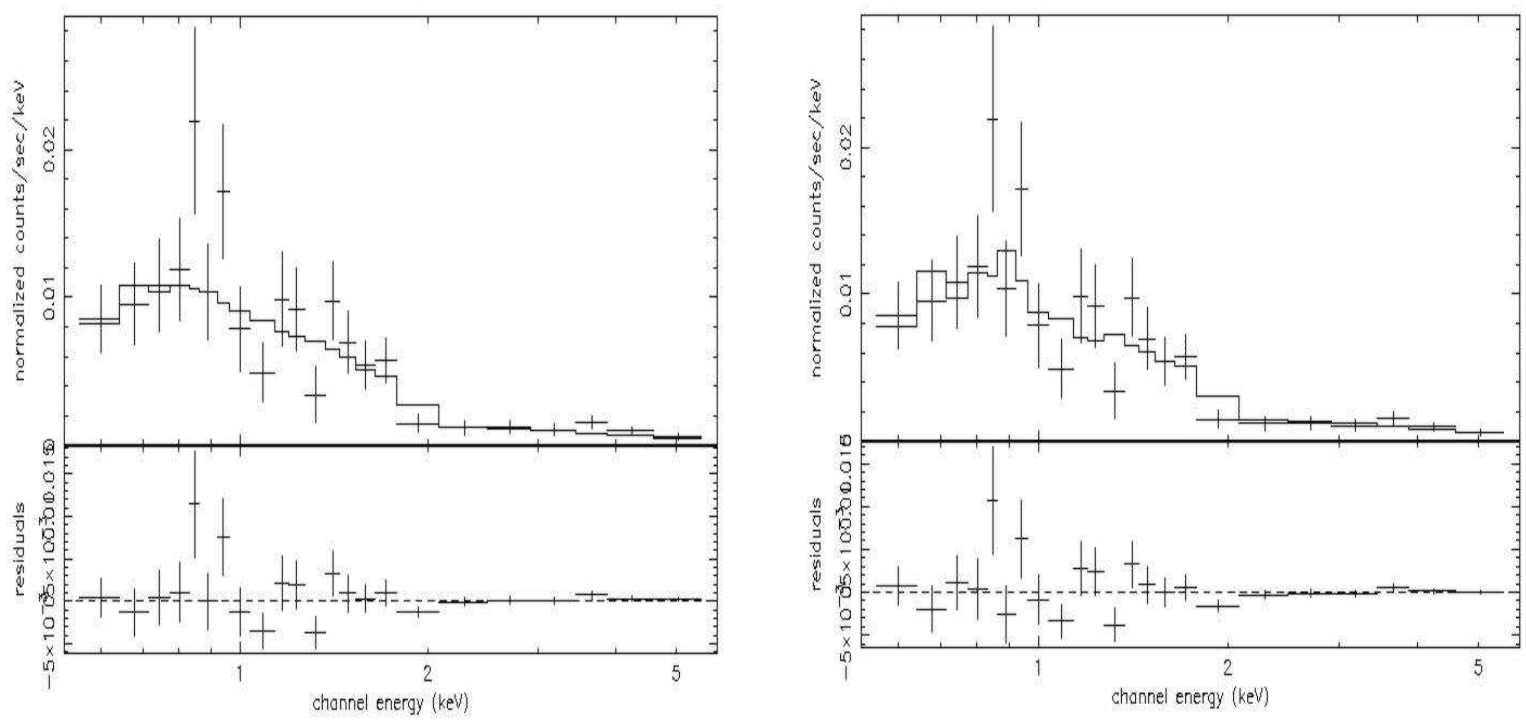

Fig. 7. - The X-ray spectrum and absorbed power law + MEKAL model fits for NGC 4435 for fixed (left) low abundance $0.1 Z_{\odot}$ and Galactic absorption and (right) solar abundance with moderate absorption, $n_{\mathrm{H}}=3.85 \times 10^{21} \mathrm{~cm}^{-2}$, inferred from optical extinction measurements.

The X-ray properties of NGC 4435 are also qualitatively explained by a collision with NGC 4438. Little X-ray emission is found in the outer regions of the optical halo in NGC 4435, but rather weak emission is concentrated in the inner nuclear regions with the soft emission, from gas and/or recent star formation, displaced from the hard emission, possibly from a low luminosity AGN, located at the galaxy's optical center. A collison with NGC 4438 would strip gas more easily from the outer regions of the smaller of the two galaxy collision partners, thus accounting for the absence of X-ray emission in the outer optical halo of NGC 4435. In galaxy collisions the gas distribution in the inner disk of the galaxy is driven toward its nucleus and distorted by the collision such that star formation is often induced offset from the galactic center. This could account for the high density $\left(4000 \mathrm{~cm}^{-3}\right)$ of $10^{4} \mathrm{~K}$ gas and younger than normal stellar distribution observed by Kenney et al. (1995) in the nuclear region of NGC 4435, its transition object classification, and the offset of the soft and hard X-ray emission peaks. Further in this scenario one would expect the pre-collision gas mass and densities in the outer disk of NGC 4435 to have been much higher than presently observed. Even given the lower limit on the densities observed today in NGC 4435 $\left(n_{e} \gtrsim 0.01 \mathrm{~cm}^{-3}\right)$, ram pressure due to the ISM-ISM interactions in a collision with NGC 4438 could dominate over ICM-ISM interations. The ram pressure on gas in NGC 4438 due to ISM-ISM interactions in a high velocity $\left(\sim 900 \mathrm{~km} \mathrm{~s}^{-1}\right)$ galaxy-galaxy collision with NGC 4435 would exceed the the ram pressure on gas in NGC 4438 due to its motion through the Virgo ICM by at least a factor of 10 . 


\subsection{Point Sources}

We identified point source candidates in the $6^{\prime} .6 \times 9^{\prime} .4$ field shown in Figure 1 using a multiscale wavelet decomposition algorithm in three energy bands: broadband $(0.3-10 \mathrm{keV})$, soft band $(0.3-$ $2 \mathrm{keV})$ and hard band $(2-10 \mathrm{kev})$. The wavelet decomposition threshold was set at 5 photons. In addition to the northwestern and southeastern outflow regions in NGC 4438 and the central region of NGC 4435, we detected 30 X-ray point sources in the broadband. These are listed in Table 5 and shown in Figure 1. Backgrounds were determined by local annuli around each source. We consider 10 source counts necessary for a flux measurement in a given bandpass. Assuming an effective photon index of 1.4 for a power law model with Galactic absorption $\left(2.64 \times 10^{20} \mathrm{~cm}^{-2}\right), 10$ source counts correspond to an absorbed flux limit in the $0.3-10 \mathrm{keV}$ band of $3.6 \times 10^{-15} \mathrm{erg} \mathrm{s}^{-1} \mathrm{~cm}^{-2}$ and limiting luminosity at $16.1 \mathrm{Mpc}$ of $1.2 \times 10^{38} \mathrm{erg} \mathrm{s}^{-1}$.

The sources in NGC 4438 are strongly clustered. Most sources lie to the south of the galactic center and either extend along the line of a bright extended X-ray filament or lie just east of the stellar tidal ridge along the leading (southeastern) edge (Kenney et al. 1995) of the galaxy. If we consider a $2^{\prime}$ circular region around the telescope aim point encompassing NGC 4438, where we expect our sample of sources with $\gtrsim 10$ photon counts to be complete, we find 8 independent sources, i.e. 6 sources in the soft band only (red), 1 source in the hard band only (cyan), and 1 source that exceeds 10 counts in both (magenta). Using the Cosmic X-ray Background (CXB) cumulative number counts curves $N(>S$ ) from the Chandra Deep Field North $1 \mathrm{Ms}$ survey (Brandt et al. 2001) combined with our flux detection limits, we would expect to see $3 \pm 1$ background sources in the soft band and $1_{-0.5}^{+1.0}$ background source in the hard band. Since NGC 4438 is known to have a significant population of old stars (Bonatto et al. 2000), we expect that most of the excess sources in NGC 4438 are low mass X-ray binaries (LMXB's). Using the $N(>S)$ curve for LMXB's in the Milky Way (Grimm et al. 2002) scaled by the ratio of the dynamical masses of the galaxies, we expect $4.8_{-0.6}^{+0.2}$ LMXB's in NGC 4438 above 10 counts and we detect 4 , consistent with this prediction. The flux corresponding to 10 counts in the hard band is, however, above the high luminosity cut-off of $2.7 \times 10^{38} \mathrm{erg} \mathrm{s}^{-1}$ for LMXB's given by those authors. The single hard source detected above the CXB prediction for this band may, if not part of the cosmic X-ray background, be a high mass X-ray binary (HMXB). Since the cumulative number distribution of HMXB's is highly correlated with the current star formation rate (Grimm et al. 2003), we can use the hard band data to place an upper limit on the current star formation rate $(s f r)$ in NGC 4438 outside of the nucleus of $s f r \lesssim 0.40_{-0.13}^{+0.24} M_{\odot} \mathrm{yr}^{-1}$. This is consistent with an estimate of the upper limit on star formation determined from FIR data, $s f r \lesssim 0.42_{-0.24}^{+0.54} M_{\odot} \mathrm{yr}^{-1}$, where we have used $\log \left(L_{\mathrm{FIR}} / L_{\odot}\right)=9.38 \pm 0.36$ from Bonatti et al. (2000) and the star formation rate FIR luminosity correlation from Grimm et al. (2002). The lack of point sources to the north of the bulge is also understandable in the context of a collision between NGC 4438 and NGC 4435. Since the strength of galaxy-galaxy tidal interactions vary significantly over galactic scales, tidal interactions from such a collision would disrupt existing stellar distributions more strongly to the north, near the point of closest approach, than to the south or in the tightly bound bulge. 
Table 5. Broadband Point Sources

\begin{tabular}{|c|c|c|c|}
\hline $\begin{array}{l}\text { RA (J2000) } \\
\text { (hh mm ss) }\end{array}$ & $\begin{array}{c}\text { Dec }(\mathrm{J} 2000) \\
(\operatorname{deg} \operatorname{arcmin} \operatorname{arcsec})\end{array}$ & $\begin{array}{l}\text { Net Source } \\
\text { Counts }\end{array}$ & $\begin{array}{c}\text { Source Radius } \\
\text { arcsec }\end{array}$ \\
\hline 122729.177 & +130356.91 & $8.4 \pm 3.4$ & 3.94 \\
\hline 122736.757 & +125839.59 & $11.1 \pm 3.6$ & 1.97 \\
\hline 122737.123 & +130440.23 & $14.9 \pm 4.5$ & 4.92 \\
\hline 122737.193 & +130121.46 & $13.1 \pm 3.9$ & 2.46 \\
\hline 122737.665 & +130036.20 & $15.7 \pm 4.4$ & 2.96 \\
\hline 122737.999 & +130451.06 & $22.7 \pm 5.5$ & 4.92 \\
\hline 122738.202 & +130158.86 & $9.2 \pm 3.5$ & 2.45 \\
\hline 122740.423 & +130456.97 & $17.7 \pm 4.5$ & 3.94 \\
\hline 122740.962 & +130455.98 & $21.7 \pm 4.8$ & 2.95 \\
\hline 122741.503 & +125820.91 & $15.6 \pm 4.1$ & 2.46 \\
\hline 122742.748 & +130002.26 & $47.9 \pm 7.1$ & 1.97 \\
\hline 122742.916 & +130235.76 & $5.0 \pm 2.7$ & 2.46 \\
\hline 122743.286 & +125958.32 & $13.0 \pm 4.0$ & 1.96 \\
\hline 122743.657 & +130025.8 & $7.8 \pm 3.0$ & 1.47 \\
\hline 122744.196 & +125833.21 & $12.9 \pm 4.1$ & 3.45 \\
\hline 122744.633 & +130025.39 & $22.6 \pm 5.1$ & 1.96 \\
\hline 122745.138 & +125943.07 & $6.4 \pm 2.9$ & 1.97 \\
\hline 122745.205 & +130019.48 & $8.1 \pm 3.2$ & 1.48 \\
\hline 122745.239 & +125912.57 & $20.1 \pm 4.7$ & 2.95 \\
\hline 122745.575 & +130042.61 & $22.3 \pm 5.8$ & 1.96 \\
\hline 122746.485 & +125929.79 & $27.6 \pm 5.4$ & 1.97 \\
\hline 122746.586 & +125907.65 & $6.2 \pm 2.7$ & 1.48 \\
\hline 122747.125 & +130021.94 & $13.4 \pm 4.0$ & 1.97 \\
\hline 122747.360 & +130045.56 & $7.8 \pm 3.0$ & 1.48 \\
\hline 122747.360 & +130101.30 & $27.6 \pm 5.8$ & 2.46 \\
\hline 122747.394 & +130053.92 & $7.0 \pm 2.9$ & 1.48 \\
\hline 122747.427 & +125914.53 & $32.1 \pm 5.8$ & 1.97 \\
\hline 122750.391 & +130414.66 & $4.3 \pm 2.2$ & 1.97 \\
\hline 122754.362 & +130118.51 & $13.9 \pm 5.3$ & 5.91 \\
\hline 122754.666 & +130318.56 & $29.9 \pm 5.6$ & 2.95 \\
\hline
\end{tabular}

Note. - The broadband energy bandpass is $0.3-10 \mathrm{keV}$. Source radii denote the radius of the circular region used to exclude the point source from the spectral analyses of the diffuse gas. 
Sources are also seen in Figure 1 to be clustered near NGC 4435. Four source candidates, in addition to the nucleus and soft X-ray peak 5." 4 to its northeast discussed previously, are identified by the algorithm. While these sources are consistent with being point sources, the broadening of the point spread function this far from the telescope aim point makes it difficult to distinguish between point sources and regions of extended emission that may be associated with the galaxy.

We find no evidence for the weak (marginally significant) X-ray emission found in Einstein HRI maps (Kotanyi et al. 1983; Fabianno et al. 1992) located roughly midway between NGC 4438 and NGC 4435 near a peak in HI emission (Hibbard \& van Gorkam 1990). Using backgrounds determined locally from several positions on the detector chips away from the galactic emission to subtract the expected Virgo cluster emission, we find that the excess X-ray flux in the $0.3-2 \mathrm{keV}$ energy band from a circular region of radius $30^{\prime \prime}$ centered at $\left(12^{h} 27^{m} 45.9^{s},+13^{\circ} 3^{\prime} 2^{\prime \prime} .2\right)$, where the marginal Einstein detection was reported, is consistent with zero in all cases. Thus assuming a thermal spectral model with $k T=0.34 \mathrm{keV}$ and Galactic absorption, we place a $3 \sigma$ upper limit on the excess flux in this region of $\lesssim 3.7 \times 10^{-15} \mathrm{erg} \mathrm{s}^{-1} \mathrm{~cm}^{-2}$. However, it should be noted that this region crosses the chip gap between S2 and S3 in our observation, so a compact source falling within the chip gap might have been missed.

\section{Conclusions}

In this paper we presented results from a $25 \mathrm{ks}$ Chandra ACIS-S observation of the peculiar spiral galaxy NGC 4438 in the Virgo Cluster taken on 29 January 2002. We were able to separately study the diffuse extended emission features and the central $1 \mathrm{kpc}$ surrounding the nuclear source. We found a remarkable correspondence between the X-ray emission features in both regions and those seen in $\mathrm{H} \alpha$ images. We also observed X-ray emission from the nearby galaxy NGC 4435 and identified point sources in both galaxies.

In summary we found:

1. The extended emission in NGC 4438 can be separated into a bulge, within a radius $\sim 2.3 \mathrm{kpc}$ from the galaxy's center, and a network of morphologically disturbed filaments with thicknesses $\sim 1 \mathrm{kpc}$ and lengths $4-10 \mathrm{kpc}$ extending out of the plane of the disk west and southwest of the bulge. These X-ray filaments correspond closely to similar features observed in $\mathrm{H} \alpha+[\mathrm{N}$ II] (Kenney et al. 1995; Keel \& Wehrle 1993; Chemin et al. 2003) with low gas velocities $\left(\lesssim 200 \mathrm{~km} \mathrm{~s}^{-1}\right)$ such that the gas may still be gravitationally bound to the galaxy.

2. The extended X-ray emitting gas associated with NGC 4438 is well fit by a single temperature MEKAL model with fixed Galactic absorption $\left(n_{H}=2.64 \times 10^{20} \mathrm{~cm}^{-2}\right)$, temperature $k T=$ $0.42_{-0.06}^{+0.07} \mathrm{keV}$, and element abundances $A=0.11_{-0.02}^{+0.05} Z_{\odot}$. The observed $0.3-2 \mathrm{keV}$ flux for the extended gas is $3.35 \times 10^{-13} \mathrm{erg} \mathrm{s}^{-1} \mathrm{~cm}^{-2}$ corresponding to an unabsorbed X-ray luminosity at $16.1 \mathrm{Mpc}$ of $1.24 \times 10^{40} \mathrm{erg} \mathrm{s}^{-1}$. We find no significant X-ray spectral differences between 
gas in the filaments and gas in the bulge.

3. Assuming simple geometries for the extended features and uniform filling of their associated volumes, we infer electron densities $\left\langle n_{e}^{2}\right\rangle^{1 / 2} \sim 0.02-0.04 \mathrm{~cm}^{-3}$ for both gas in the filaments and the bulge, and cooling times $t_{\text {cool }} \sim 4-7 \times 10^{8} \mathrm{yr}$. The total mass of hot gas in a $95^{\prime \prime}$ aperture surrounding the galaxy is $M \lesssim 3.7 \times 10^{8} M_{\odot}$.

4. The low temperatures $\left(0.4 \mathrm{keV}\right.$ or $\left.4.6 \times 10^{6} \mathrm{~K}\right)$ and low velocities observed in the extended features, as well as the asymmetrically disturbed morphology and strong correlation between $\mathrm{H} \alpha$ and X-ray emitting gas, are most naturally explained by tidal interactions and multiphase ISM-ISM interactions, acting during a galaxy-galaxy collision that occurred $\sim 10^{8}$ years ago, as NGC 4438 passed $\sim 5 \mathrm{kpc}$ to the south of NGC 4435's center with relative velocity $\sim 900 \mathrm{~km} \mathrm{~s}^{-1}$.

5. X-ray emission in the center of NGC 4438 is observed from two regions corresponding to the two outflow shells observed in $\mathrm{H} \alpha+[\mathrm{N} \mathrm{II}]$ with HST by Kenney \& Yale (2002) and to the two peaks observed in the radio continuum by Hummel \& Saikia (1991). The brighter feature extends $\sim 360 \mathrm{pc}$ to the northwest of the optical nucleus with the peak of the Xray emission $\lesssim 0^{\prime \prime} .7$ from the compact nuclear source. The fainter southeastern outflow shell consists of patchy, shell-like emission in a circular aperture of radius $\sim 130 \mathrm{pc}$ located $730 \mathrm{pc}$ southeast of the nuclear source. The X-ray spectrum of the northwest outflow region, including the compact nuclear source, is well fit by an absorbed MEKAL model thermal component with temperature $k T=0.58_{-0.10}^{+0.04} \mathrm{keV}$, abundance $0.14 Z_{\odot}$, and absorbing column $n_{H}=1.9_{-0.04}^{+0.10} \times 10^{21} \mathrm{~cm}^{-2}$ and a heavily obscured $\left(n_{H}=2.9_{-2.0}^{+3.1} \times 10^{22} \mathrm{~cm}^{-2}\right)$ AGN-like (photon index fixed at 2.0) nuclear source.

6. The $0.3-2 \mathrm{keV}(2-10 \mathrm{keV})$ unabsorbed X-ray luminosities from the northwest outflow region are $1.02 \times 10^{40} \mathrm{erg} \mathrm{s}^{-1}\left(2.6 \times 10^{38} \mathrm{erg} \mathrm{s}^{-1}\right)$ for the thermal component and $2.4 \times 10^{39} \mathrm{erg} \mathrm{s}^{-1}(2.0 \times$ $10^{39} \mathrm{erg} \mathrm{s}^{-1}$ ) for the non-thermal component, with large uncertainties in the latter due to uncertainties in the absorption correction. Assuming the same spectral model for the gas in the southeastern outflow bubble, the inferred electron densities and cooling times in both outflow bubbles are $0.5 \mathrm{~cm}^{-3}$ and $3 \times 10^{7} \mathrm{yr}$. The total amount of hot gas in the northwest outflow region is $\sim 3.5 \times 10^{6} M_{\odot}$. The spectral properties and extent of the outflow features are consistent with gas heated by shocks driven into the surrounding ISM with shock velocities $\gtrsim 600 \mathrm{~km} \mathrm{~s}^{-1}$ by nuclear outbursts $1-2 \times 10^{6}$ years ago. The large amount of kinetic energy carried in the outflow gas $\left(\gtrsim 3.5 \times 10^{54}\right.$ ergs for the northwestern outflow bubble), coupled with low upper limits $\left(\lesssim 0.1 M_{\odot} \mathrm{yr}^{-1}\right)$ on recent star formation in the nucleus of NGC 4438, favor an obscured AGN, over a compact starburst, as the nuclear source.

7. Asymmetric X-ray emission is observed in a $2.5 \mathrm{kpc}$ circular aperture about the optical center of NGC 4435 with soft $(0.4-2 \mathrm{keV})$ and hard $(2-10 \mathrm{keV})$ band total observed fluxes of $6.5 \times 10^{-14} \mathrm{erg} \mathrm{s}^{-1} \mathrm{~cm}^{-2}$ and $8.1 \times 10^{-14} \mathrm{erg} \mathrm{s}^{-1} \mathrm{~cm}^{-2}$. The peak of the hard emission occurs at the optical center of the galaxy; while the peak of the soft emission is displaced $416 \mathrm{pc}$ to 
the northeast. The spectrum is consistent with a dominant AGN nuclear source with photon index $\Gamma \sim 1.6-2$ and a weaker, $0.2-0.3 \mathrm{keV}$ extended thermal component. For fixed $0.1 Z_{\odot}$ abundance and Galactic absorption, we infer an electron density $n_{e} \sim 0.015 \mathrm{~cm}^{-3}$ and, assuming a uniform filling factor, an X-ray gas mass $M \sim 3 \times 10^{7} M_{\odot}$. However, the electron density and X-ray emitting gas mass are sensitive to the assumed element abundance (decreasing by a factor $\sim 3$, as the abundance is varied from $0.1-1.0 Z_{\odot}$ ) and absorbing column (increasing by as much as a factor of 5 , given optical values for the extinction) and thus are highly uncertain. The fact that gas is primarily concentrated near the nucleus is consistent with much of the outer gas having been stripped from NGC 4435, the smaller of the two galaxies, during a collision with NGC 4438.

8. Point sources, identified above the broadband $(0.3-10 \mathrm{keV})$ flux (luminosity) limit of $3.6 \times$ $10^{-15} \mathrm{erg} \mathrm{s}^{-1} \mathrm{~cm}^{-2}\left(1.2 \times 10^{38} \mathrm{erg} \mathrm{s}^{-1}\right)$, are found asymmetrically clustered mainly to the south of the galactic center in NGC 4438 consistent with the stars north of the galactic center having been disrupted in a collision with NGC 4435. Excess sources above the Cosmic X-ray Background prediction in the soft band are consistent with the expected number of LMXB's based on the mass of the galaxy. The single hard band source places an upper limit on the current star formation rate outside the nucleus of $\lesssim 0.4_{-0.13}^{+0.24} M_{\odot} \mathrm{yr}^{-1}$.

This work has been supported in part by NASA contract NAS8-39073, and the Smithsonian Institution. MEM also acknowledges support from the Radcliffe Institute for Advanced Study at Harvard University. This work has made use of the NASA/IPAC Extragalactic Database (NED)

which is operated by the Jet Propulsion Laboratory, California Institute of Technology, under contract with the National Aeronautics and Space Administration. We wish to thank Sebastian Heinz, Ralph Kraft, Paul Nulsen, and Laurent Chemin for useful discussions.

\section{REFERENCES}

Abadi, M.G., Moore, B. \& Bower, R.G. 1999, MNRAS, 308, 947

Argyle, R.W. \& Clements, E.D. 1990, Observatory, 110, 93A

Balsara, D., Livio, M. \& O’Dea, P. 1994, ApJ, 437, 83

Bautz, M.W., et al. 1998, X-ray Optics, Instruments and Missions, ed. R.B. Hoover \& A. B. Walker, Proc. SPIE, 3444, 210

Bekki, K., Couch W.J. \& Shioya, Y. 2002, ApJ, 577, 651

Bicknell, G.V., Dopita, M.A., Tsvetanov, Z.I., \& Sutherland, R.S. 1998, ApJ, 495, 680

Binney, J. \& Merrifield, M. 1998, Galactic Astronomy (Princeton University Press, Princeton), p. 137

Bonatto, C., Bica, E., Pastoriza, M.G. \& Alloin, D. 2000, A \& A, 355, 99 
Brandt, W.N., Alexander, D.M., Hornschemeier, A.E., Garmire, G.P., Schneider, D.P., Barger, A.J., Bauer, F.E., Broos, P.S., Cowie, L.L., Towenlsey, L.K., Burrows, D.N., Chartas, G., Feigelson, E.D., Griffiths, R.E., Nousek, J.A., \& Sargent, W.L.W 2001, ApJ, 122, 2810

Butcher, H. \& Oemler, A. Jr 1978, ApJ, 219, 18

Butcher, H. \& Oemler, A. Jr 1984, ApJ, 285,426

Byrd, G. \& Valtonen, M. 1990, ApJ, 350, 89

Canizares, C.R., Fabbiano, G., \& Trinchieri, G. 1987, ApJ, 387, 484

Cayatte, V., van Gorkom, J.H., Balkowski, C. \& Kotanyi, C. 1990, AJ, 100, 604

Cecil, G., Bland-Hawthorn, J., Veilleux, S. 2002, ApJ, 576, 745

Chemin, L., Cayatte, V., Balkowski, C., Amram, P., Marcelin, M., Garrido, O., Boulesteix, J., Carignan, C., Boselli, A., Vollmer, B., Adami, C. \& Hernandex, O. 2004, in IAU Symposium 217: Recycling Intergalactic and Interstellar Matter, ASP Conference Series, Vol. XXX ed. P.-A Duc, J. Braine \& E. Brinks, in press, preprint astroph/0309496

Chincarini, G. \& de Souza, R. 1985, A \& A, 153, 218

Combes, F., Dupraz, C., Casoli, F., \& Pagani 1988, A \& A, 203, L9

Couch, W.J., Barger, A.J., Smail, I., Ellis, R.S. \& Sharples, R.M. 1998, ApJ, 497, 188

Di Matteo, T., Allen, S.W., Fabian, A.C., Wilson, A.S., Young, A.J. 2003, ApJ, 582, 133

Dressler, A. \& Gunn, J. 1983, ApJ, 270, 7

Dressler, A.,Oemler, A., Butcher, H. \& Gunn, J. 1994, ApJ, 430, 107

Ellingson, E., Lin, H., Yee, H.K.C \& Carlberg, R.G. 2001, ApJ, 547, 609

Fabbiano, G., Kim, D.-W. \& Trinchieri, G. 1992, ApJS, 80, 531

Fabian, A.C. \& Rees, M.J. 1995, MNRAS, 277, 55

Ferrarese, L. \& Merritt, D. 2000, ApJ, 539, L9

Freeman, P.E., Kashyap, V., Rosner, R., \& Lamb, D.Q. 2002, ApJS, in press, preprint astro-ph 0108429

Fujita, Y. \& Nagashima, M. 1999, ApJ, 516, 619

Garmire, G.P. et al. 1992, AIAA, Space Programs and Technologies Conference, Huntsville, AL, March $24-27$

Gebhardt, K., Bender, R., Bower, G, Dressler, A., Faber, S.M., Filippenko, A.V., Green, R.,Grillmair, C., Ho, L.C., Kormendy, J., Lauer, T.R., Magorrian, J, Pinkney, J., Richstone, D., Tremaine, S. 2000, ApJ, 539, L13

Grimm, H.-J., Gilfanov, M. \& Sunyaev, R. 2003, MNRAS, 339, 793

Grimm, H.-J., Gilfanov, M. \& Sunyaev, R. 2002, A \& A, 391, 923

Gunn, J., \& Gott, J. 1972, ApJ, 176, 1

Halderson, E., Moran, E.D., Filippenko, A.V. \& Ho, L.C. 2001, AJ, 122, 637 
Hibbard, J.E. \& van Gorkom, J.H. 1990 in The Interstellar Medium in External Galaxies: Summaries of Contributed Papers, ed. D.J. Hollenbach \& H.A. Thronson, Jr. (Washington:NASA), 214

Ho, L.C., Filippenko, A.V. \& Sargent, W.L.W. 1997, ApJS, 112, 315

Ho, L.C., Filippenko, A.F., Sargent, W.L.W., \& Peng, C.Y. 1997b, ApJS, 112, 391

Ho, L.C., Filippenko, A.F., \& Sargent, W.L.W. 1997c, ApJ, 487, 568

Hollenbach, D. \& McKee, C.F. 1979, ApJS, 41, 555

Hummel, E. \& Sakai, D.J. 1991, A \& A, 249, 43

Hummel, E., van Gorkom, J.H. \& Kotanyi, C.G. 1983, ApJ, 267, L5

Keel, W.C. \& Wehrle, A.E. 1993, AJ, 106, 236

Kelson, D.D., Illingworth, G.D., Tonry, J.L., Freedman, W.L., Kennicutt, R.C. Jr., Mould, J.R., Graham, J.A., Huchra, J.P., Machri, L.M., Madore, B.F., Ferrarese, L., Gibson, B.K., Sakai, S., Stetson, P.B., Ajhar, E.A., Blakeslee, J.P., Dressler, A., Ford, H.D., Hughes, S.M.G., Sebo, K.M., \& Silberman, N.A. 2000, ApJ, 529, 768

Kenney, J.D.P., Rubin, V.C., Planesas, P. \& Young, J.S. 1995, ApJ, 438, 135

Kenney, J.D.P. \& Yale, E.E. 2002, ApJ, 567, 865

Koopman, R.A. \& Kenney, J.D.P. 2002, ApJ, submitted, preprint astro-ph/0209547

Koratkar, A., Dewtra, S.E., Heckman, T., Filippenko, A.V., Ho, L.C. \& Rao, M. 1995, ApJ, 440, 132

Kotanyi, C., van Gorkom, J.H. \& Ekers, R.D. 1983, ApJ, 273, L7

Lavery, R. \& Henry, J.P. 1988, ApJ, 330, 596

Lavery, R., Pierce, M. \& McClure, R. 1992, AJ, 104, 2067

Miller, R.H. 1986, A \& A, 167, 41

Miller, N. in Carnegie Obseratories Astrophysics Series, Vol.3: Clusters of Galaxies: Probes of Cosmological Structure and Galaxy Evolution, ed. J.S. Mulchaey, A. Dressler, \& A. Oemler (Pasadena; Carnegie Observatories, 2003), preprint astro-ph/0303393

Moore, B., Katz, N., Lake, G., Dressler, A., \& Oemler, A. 1996, Nature, 379, 613

Müller, E., Mair, G., \& Hillebrandt, W. 1989, A \& A, 216, 19

Narayan, R. \& Yi, I. 1995, ApJ, 452, 710

Nulsen, P.J.E. 1982, MNRAS, 198, 1007

Ohashi, T., Kikuchi, K., Matsushita, K., Yamasaki, N.Y., Kushino, A. \& ASCA Virgo Project Team 1998, in The Hot Universe, Proceedings of the IAU Symposium no. 188, eds. K. Koyama, S. Kitamoto, \& M. Itoh (Dordrecht:Kluwer Academic, 1998), 317

Osterbrock, D.E. 1989, Astrophysics of Gaseous Nebulae and Active Galactic Nuclei (University Sciece Books, Mill Valley, CA) p. 134

Pellegrini, S., Baldi, A., Fabbiano, G. \& Kim, D.-W. 2003, ApJ, in press, preprint astro-ph/0307142 
Poggianti, B.M., Smail,I., Dressler, A., Couch, W.J., Barger, A.J., Butcher, H., Ellis, R.S., \& Oemler, A. 1999, ApJ, 518, 576

Quilis,V., Moore, B., \& Bower, R. 2000, Science, 288, 1617

Roberts, T.P. \& Warwick, R.S. 2000, MNRAS, 315, 98

Schindler, S., Binggeli, B., Böhringer, H., A \& A, 343, 420

Schulz, S. \& Struck, C. 2001, MNRAS, 328, 185

Terashima, Y., Ho, L.C. \& Ptak, A.F. 2000, ApJ, 539, 161

Terashima, Y., Iyomoto, N., Ho, L.C. \& Ptak, A.F. 2002, ApJS, 139, 1

Terashima, Y. \& Wilson, A.S. 2001, ApJ, 560, 139

Veilleux, S., Cecil, G., Bland-Hawthorne, J., Tully, R.B., Filippenko, A.V., \& Sargent, W.L.W. 1994, ApJ, 433,48

Vollmer, B., Cayatte, V. Balkowski, C., \& Duschl, W.J. 2001, ApJ, 561, 708

Ward, M.J., Done,C., Fabian, A.C., Tennant, A.F., \& Shafer, R.A. 1988, ApJ, 324, 767 